\title{
First supermodule of the MACRO detector at Gran Sasso
}

\author{
MACRO Collaboration
}

S. Ahlen ${ }^{\text {d }}$, M. Ambrosio ${ }^{\mathrm{m}}$, R. Antolini ${ }^{\mathrm{c}}$, G. Auriemma ${ }^{\mathrm{o}, 2}$, A. Baldini ${ }^{\mathrm{n}}$, G.C. Barbarino ${ }^{\mathrm{m}}$, B. Barish ${ }^{\mathrm{e}}$, G. Battistoni ${ }^{\mathrm{g}}$, R. Bellotti ${ }^{\mathrm{a}}$, C. Bemporad ${ }^{\mathrm{n}}$, P. Bernardini ${ }^{\mathrm{k}}$, H. Bilokon ${ }^{\mathrm{g}}$, V. Bisi ${ }^{\mathrm{r}}$, C. Bloise ${ }^{\mathrm{g}}$, A. Boiano ${ }^{\mathrm{m}}$, C. Bower ', S. Bussino ${ }^{\circ}$, F. Cafagna ${ }^{\mathrm{a}}$, M. Calicchio ${ }^{\mathrm{a}}$, P. Campana ${ }^{g}$, D. Campana ${ }^{\mathrm{m}}$, A. Candela ${ }^{\mathrm{m}}$, M. Carboni ${ }^{\mathrm{g}}$, S. Cecchini ${ }^{\mathrm{c}}$, F. Cei ${ }^{\mathrm{o}, \mathrm{m}}$, V. Chiarella ${ }^{g}$, C. Chiera ${ }^{\circ}$, A. Cobis ${ }^{g}$, R. Cormack ${ }^{d}$, A. Corona ${ }^{\circ}$, D. Cosson ${ }^{g}$, S. Coutu ${ }^{\text {, }}$, I. D’Antone ${ }^{\text {c }}$, G. De Cataldo ${ }^{a}$, C. De Marzo a , U. Denni ${ }^{\mathrm{g}}$, N. De Seriis ${ }^{\circ}$, M. DeVincenzi ${ }^{\circ}$, A. Di Credico ', E. Diehl ', R. Diotallevi o , O. Erriquez a , C. Favuzzi a , D. Ficenec ${ }^{\text {d,3 }}$, C. Forti ${ }^{\text {g }}$, L. Foti ${ }^{o}$, A. Frani ${ }^{\text {h }}$, P. Fusco ${ }^{a}$, F. Gherarducci ${ }^{n}$, G. Giacomelli ${ }^{\text {, }}$, G. Giannini ${ }^{\text {n,1 }}$, N. Giglietto ${ }^{\text {a }}$, P. Giubellino ${ }^{\mathrm{r}}$, M. Grassi ${ }^{\mathrm{n}}$, P. Green ${ }^{\mathrm{p}}$, A. Grillo ${ }^{\mathrm{g}}$,

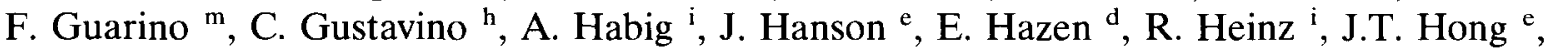
E. Iarocci ${ }^{\text {g,5 }}$, E. Katsavounidis ${ }^{\text {e }}$, E. Kearns ${ }^{\text {d }}$, S. Klein ${ }^{\text {d,4 }}$, S. Kyriazopoulou e ${ }^{\text {, E. Lamanna }}{ }^{\text {, }}$, C. Lane ${ }^{\text {f }}$, C. Lee ', D. Levin ', P. Lipari ${ }^{\circ}$, G. Liu ${ }^{\text {e }, ~ R . ~ L i u ~}{ }^{\text {e }}$, M.J. Longo ', G. Ludlam ${ }^{\mathrm{d}}$, G. Mancarella ${ }^{\text {k, }}$, G. Mandrioli ${ }^{\text {c }}$, A. Marin ${ }^{\text {d }}$, A. Marini ${ }^{\text {g }}$, D. Martello ${ }^{k}$, G. Martellotti ${ }^{\text {, }}$ A. Marzari Chiesa ${ }^{\text {r }}$, M. Masera ${ }^{\text {r }}$, P. Matteuzzi ${ }^{\text {c }}$, A. Mengucci ${ }^{g}$, S. Merritt ${ }^{d}$, D.G. Michael ${ }^{\text {e }}$, L. Miller ', P. Monacelli ', M. Mongelli a, M. Monteno ', S. Mufson ', J. Musser ', S. Nutter ', C. Okada d, G. Osteria ${ }^{\mathrm{m}}$, O. Palamara ${ }^{\mathrm{k}}$, P. Parascandalo ${ }^{\mathrm{m}}$, S. Parlati ${ }^{\mathrm{h}}$, V. Patera ${ }^{\mathrm{g}}$, L. Patrizii ${ }^{\mathrm{c}}$, R. Pazzi ${ }^{n}$, C.W. Peck ${ }^{\mathrm{e}}$, G. Pellizzoni ${ }^{\circ}$, M. Perchiazzi ${ }^{\mathrm{a}}$, J. Petrakis ${ }^{b}$, S. Petrera ${ }^{k}$, N.D. Pignatano ${ }^{\text {, }}$, C. Pinto ${ }^{\text {k, P. Pistilli }}{ }^{k}$, F. Predieri ${ }^{\text {, }}$, L. Ramello ${ }^{r}$, J. Reynoldson ${ }^{\text {h }}$, F. Ronga ${ }^{\text {g, G. Rosa }}{ }^{\circ}$, A. Sacchetti ${ }^{\text {a }}$, G. Sanzani ${ }^{\text {c }}$, C. Satriano ${ }^{o, 2}$, L. Satta ${ }^{\text {g.5 }}$, E. Scapparone ${ }^{c}$, K. Scholberg e , A. Sciubba ${ }^{0,5}$, P. Serra Lugaresi ${ }^{c}$, M. Severi ${ }^{\circ}$, M. Sitta ${ }^{r}$, P. Spinelli a , M. Spinetti ${ }^{\text {g }}$, M. Spurio ${ }^{\text {c }}$, J. Steele ${ }^{\text {e }}$, R. Steinberg ${ }^{\text { }}$, J.L. Stone ${ }^{d}$, L.R. Sulak ${ }^{d}$, A. Surdo ${ }^{k}$, G. Tarlé ${ }^{1}$, A. Tazzioli ${ }^{n}$, V. Togo ${ }^{c}$, V. Valente ${ }^{\text {g }}$, G.R. Verdone ${ }^{\mathbf{o}, 2}$, C.W. Walter ${ }^{\mathrm{c}}$, R. Webb ${ }^{\mathrm{q}}$, F. Welte ${ }^{\mathrm{d}}$ and W. Worstell ${ }^{\mathrm{d}}$

${ }^{a}$ Dipartımento di Fisica dell'Università di Barı and INFN, I-70126, Barı, Italy

${ }^{b}$ Bartol Research Institute, University of Delaware, Newark, DE 19716, USA

' Dipartimento di Fisica dell'Universtì di Bologna and INFN, I-40126, Bologna, Italy

${ }^{d}$ Department of Phystcs, Boston University, Boston, MA 02215, USA

' Department of Physics, California Institute of Technology, Pasadena, CA 91125, USA

${ }^{f}$ Department of Physics, Drexel University, Philadelphia, PA 19104, USA

${ }^{g}$ Laboratori Naztonali di Frascatt dell'INFN, I-00044, Frascati, Italy

${ }^{h}$ Laboratori Nazionali del Gran Sasso dell'INFN, 1-67010, Assergi, Italy

'Department of Physics and Astronomy, Indiana University, Bloomington, IN 47405, USA

'Dipartimento di Fisica dell'Università dell'Aquila and INFN, I-67100, L'Aquila, Italy

${ }^{k}$ Dipartımento di Fisica dell'Università di Lecce and INFN, I-73100, Lecce, Italy

'Department of Physics, Unversity of Michigan, Ann Arbor, MI 48109, USA

${ }^{m}$ Dipartimento de Fisica dell'Università di Napoli and INFN, I-80125, Naples, Italy

${ }^{n}$ Dipartimento di Fisica dell'Untversità di Pisa and INFN, I-56010, Pisa, Italy

${ }^{\circ}$ Dipartimento de Fistca dell'Università di Roma and INFN, I-00185, Roma, Italy

1 Also at Università di Trieste, I-34100 Trieste, Italy.

2 Also at Università della Basilicata, I-85100 Potenza, Italy.

${ }^{3}$ Now at Physics Department, Washington University, St. Louis, MO 63130, USA.

\footnotetext{
${ }^{4}$ Now at Department of Physics, University of California, Santa Cruz, CA 95064, USA.

5 Also at Dipartimento di Energetica, Università di Roma, Italy.
} 
"Ditision 9321, Sandia National Laboratory, Albuquerque, NM 87185, USA

${ }^{9}$ Department of Physics, Texas A\&M Unwersity, College Station, TX 77843, USA

${ }^{r}$ Dipartımento di Fisica Sperimentale di Torino and INFN, I-10125, Turth, Italy

Recelved 23 June 1992

In this paper the design, construction and performance of the lower part of the first supermodule of the MACRO detector is described.

\section{Introduction}

The MACRO experiment belongs to a new generation of underground detectors that are made possible by the large experimental halls of the Laboratori Nazionali del Gran Sasso (LNGS) of the Istituto Nazionale Fisica Nucleare (INFN). When completed, the detector will have a large acceptance $\left(\approx 10000 \mathrm{~m}^{2}\right.$ sr) for isotropic fluxes of penetrating particles and its large area will provide high sensitivity in the search of rare phenomena in the cosmic radiation.

One of the main physics goals of the MACRO experiment is to search for magnetic monopoles with the properties predicted by grand unified theories (GUT monopoles) [1] with a sensitivity well below the astrophysical bounds. The key design philosophy of MACRO for the detection of magnetic monopoles is to provide a large, highly redundant detection system allowing multiple signatures of the rare events since no more than a few magnetic monopoles could be expected in a year. Three independent and complementary methods are employed for monopole detection: scintillators, streamer tubes operated with a helium gas mixture, and plastic track-etch detectors. These provide a wide coverage of possibilities for the unknown velocity and ionization properties of monopoles. Independent triggering schemes as well as specialized custom electronics are used to insure multiple signatures and cross-checks for all possible candidate events.

The large acceptance of the detector and its design features will allow a search with good sensitivity for astrophysical point sources, such as Cygnus X-3, which may be emitting high energy gammas, neutrinos or other neutral particles. High resolution tracking of penetrating particles by the streamer tube system allows accurate pointing in celestial coordinates, $\leq 1^{\circ}$, limited primarily by multiple coulomb scattering in the rock overburden. Fast timing and $\mathrm{d} E / \mathrm{d} x$ measurements have been incorporated in the scintillator system to provide broad capabilities. In particular, upwardgoing particles are distinguished from downward-going particles at the level of $1 / 10^{7}$. Absolute time measurements can be made with accuracy $\sim 1 \mu \mathrm{s}$. Thus, MACRO has extensive capabilities for astrophysical observations.
The large scintillator mass $(\approx 600$ tons in the six supermodule configuration) of MACRO allows searches for neutrino bursts emitted in gravitational stellar collapses. The sensitivity of scintillator to low energy interactions is well established. Hence for this purpose, MACRO employs specially designed triggers and electronics to recognize, buffer, and record a large number of low energy events over a short time period which may be an indication of a supernova. A monitoring system is now in operation that sounds an alarm (via modem and cellular phone) to provide immediate indication if a candidate stellar collapse event has occurred.

The investigation of the standard penetrating cosmic radiation with high statistics will allow systematic studies on the primary cosmic ray composition. The MACRO tracking system covers a significantly larger area than any other detector operated deep underground. It is possible to distinguish two or more muons that are within a few centimeters of each other as well as those which are separated up to $\sim 75 \mathrm{~m}$. Many large separation multiple muon events have already been observed along with several very high multiplicity $(>40$ muons) events. As statistics are accumulated, these data will allow for the most sensitive studies to date of the primary cosmic ray composition in the ultra high energy (UHE) range.

A unique feature of the MACRO detector is the possibility of studying events in coincidence with the extensive air shower detector EAS-TOP, located on top of the Gran Sasso mountain. For the events passing through both detectors the primary energy is measured by EAS-TOP and the underground high energy $(\geq 2 \mathrm{TeV}$ ) muon content is measured by MACRO. These data are identified by coincidences given by our common universal clock. This capability allows primary cosmic ray composition studies that select a higher energy region than underground muons alone in which the statistics favor lower energies.

In this paper we describe the design, construction, and performance of the lower part of the first supermodule of the MACRO detector. It was brought into operation in February 1989, and, except for a three month interval in the summer of 1989 , was in continuous operation. The principal purpose of this operation 


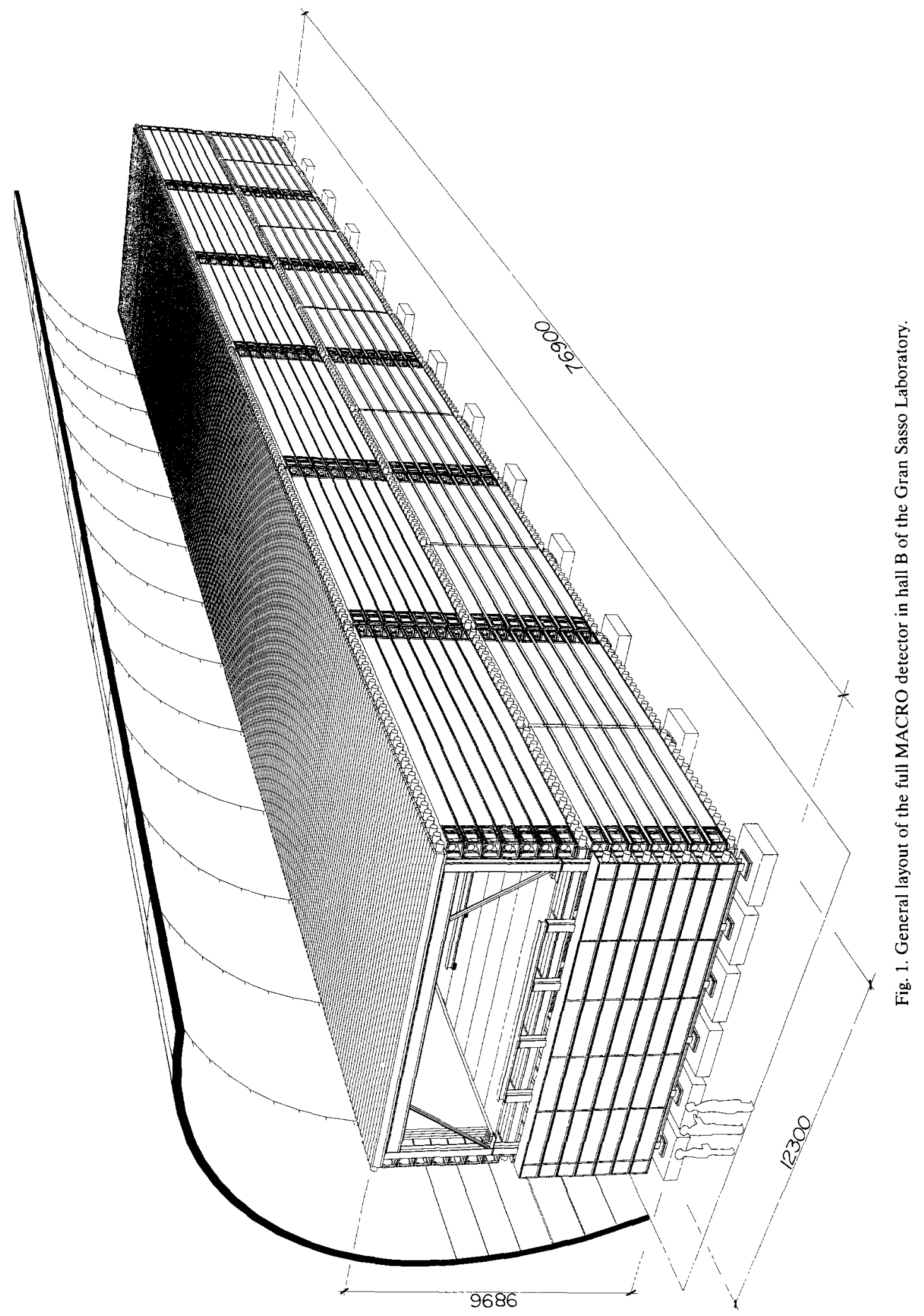


has been to test the MACRO design principles and therefore to guide the further development of the apparatus. In general, the original design has proven sound and construction is proceeding with few modifications relative to what is described in this paper. The other five supermodules of the detector are already built and are currently being put into operation.

\section{General layout of the MACRO detector}

The general layout of final MACRO detector is shown in fig. 1. It consists of six "supermodules" with overall dimensions of $12 \mathrm{~m} \times 77 \mathrm{~m} \times 9 \mathrm{~m}$. Each supermodule has a lower part of $4.8 \mathrm{~m}$ height and an upper part that will bring the total height to $9 \mathrm{~m}$. In this paper we describe in detail the lower part of the first supermodule. Fig. 2 shows a cross-sectional end view of the lower part of a MACRO supermodule. The lower part of a supermodule is made of two modules each with horizontal area of $6 \mathrm{~m} \times 12 \mathrm{~m}$. Between the two modules there is a gap of $0.3 \mathrm{~m}$ with no sensitive element since it is occupied by the supporting structure of the apparatus. The sides and north face of supermodule 1 are covered with liquid scintillation counters ${ }^{\# 1}$. There are two types of scintillator units, those for the horizontal planes and those for the verti-

\#1 Sandwiched between six planes of limited streamer tubes. cal planes. The scintillator system and its electronic instrumentation are described below in section 3. Each layer of the horizontal scintillator counters is sandwiched between two planes of limited streamer tubes, each $12 \mathrm{~m}$ long with a cross-sectional area of $3 \mathrm{~cm} \times 3$ $\mathrm{cm}$. There are six more horizontal planes of streamer tubes in between, for a total of ten planes. The eight inner planes are separated by passive absorbers (iron and rock $\sim 60 \mathrm{~g} / \mathrm{cm}^{2}$ ) in order to set a minimum threshold $\sim 1 \mathrm{GeV}$ for vertical muons crossing the detector. The horizontal streamer tube planes are instrumented with external pick-up strips which make an angle of $26.5^{\circ}$ with the streamer tubes' wires and provide a stereo readout of track hits. The streamer tube system and its electronics are described below in section 4. The active systems of scintillators and streamer tubes are supplemented by one horizontal layer of passive track-etch detector to provide GUT monopole detection redundancy and further ionization information about any monopole which may be detected The final configuration of MACRO will have the north and east vertical faces of the detector covered with tracketch modules. Each track-etch module consists of two types of plastic with different sensitivities separated by an aluminum sheet. The design allows it to distinguish heavily ionizing nuclear fragments from the expected signature for magnetic monopoles. This system is described in section 5. Sections 6 and 7 describe the on-line data acquisition system and the off-line data analysis environment, respectively.

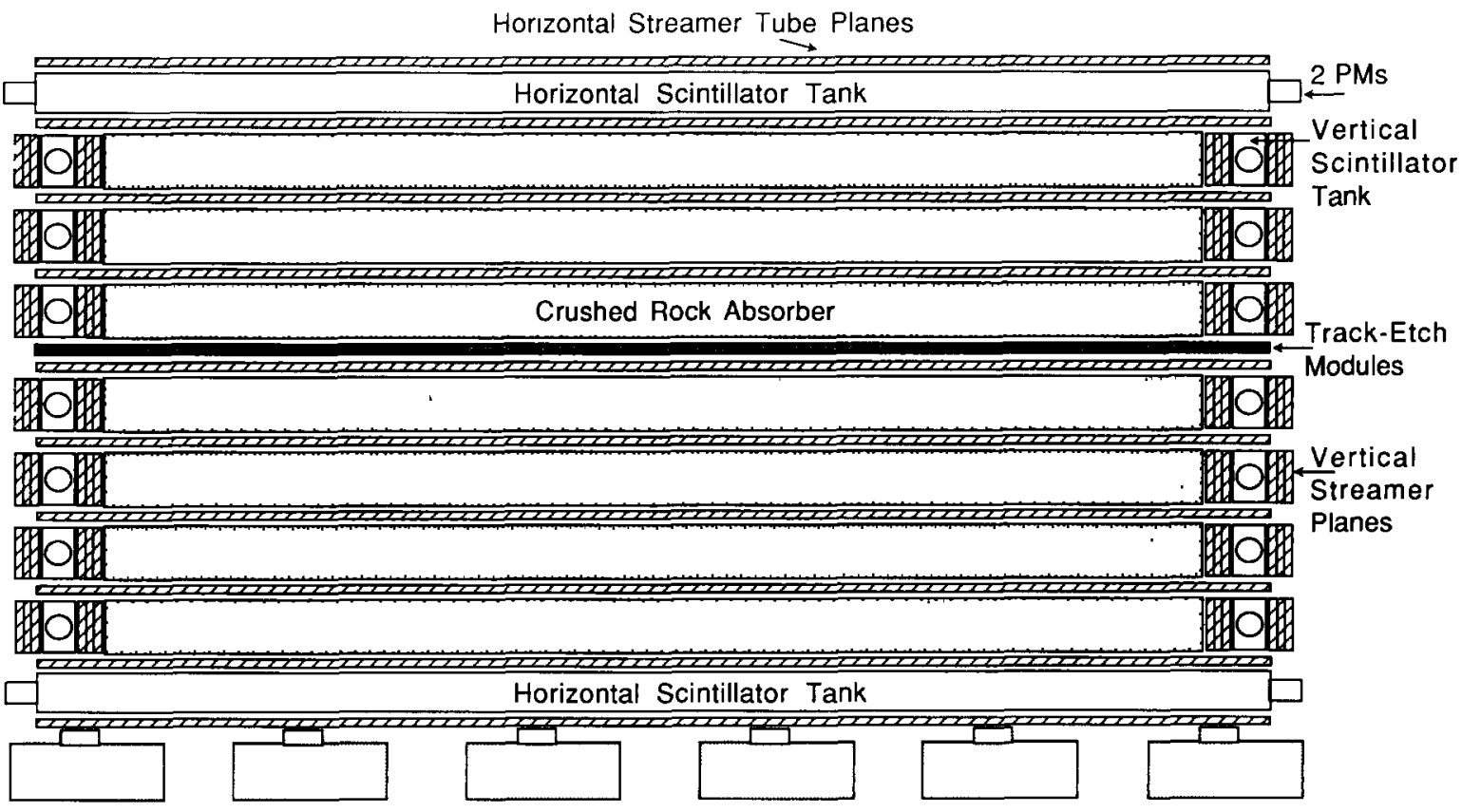

Fig. 2. Cross-sectional end view (not to scale) of the lower part of a MACRO supermodule 


\section{The scintillator system}

The MACRO scintillator system for the lower part of the first supermodule consists of 32 horizontal counters and 21 vertical counters. All are constructed from $0.63 \mathrm{~cm}$ thick PVC sheets. The horizontal counters cover two $12 \mathrm{~m} \times 12 \mathrm{~m}$ planes separated vertically by $4.8 \mathrm{~m}$ and the vertical counters cover three of the four vertical faces of this volume. The nominal length of each counter is $12 \mathrm{~m}$ of which about $11 \mathrm{~m}$ is filled with a highly transparent mineral oil-based scintillator. The composition of the scintillator oil has been optimized for this counter geometry and it is described later. Each end of the horizontal counters contains reflectors directing the scintillation light to two $20 \mathrm{~cm}$ diameter photomultipliers (PM). (Note: Hamamatsu R-1408 tubes are used in the first supermodule; however, EMI D-642 tubes will be used for the remainder of the MACRO detector.) The anodes and one dynode of the pair of PMs are connected separately in parallel providing two PM signals from each counter end. The vertical counters are similar except that each end is instrumented with just one PM.

The output PM signals from each counter end (a total of 212 signals since both anode and dynode signals are used) are fed to fanouts that make copies for driving all the subsequent triggering and measurement electronics. This electronic system provides a collection of triggers with overlapping sensitivities. Included are triggers specially designed for magnetic monopoles passing through the detector with velocities as slow as $1.3 \times 10^{-4} c$ as well as others for muons and bursts of low energy neutrinos from supernovae. It also provides redundant measurements of the time structure of the signals, their charge, and time of occurrence.

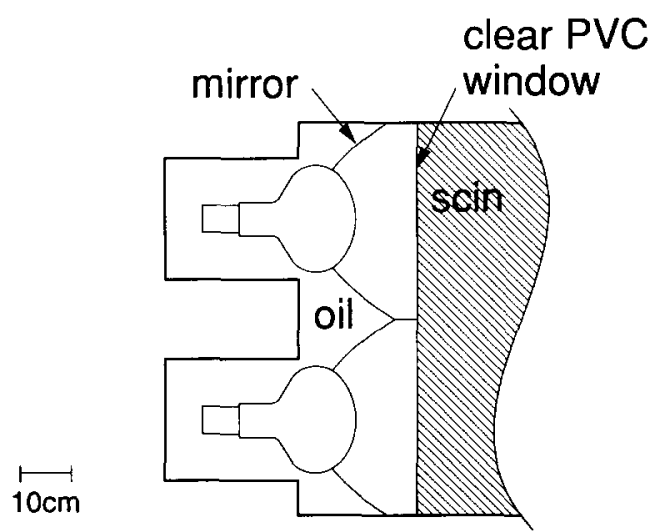

Fig. 3. Details of each end of the horizontal scintillation counters. The anodes and one dynode of the two phototubes are connected separately in parallel. The length of the sensitive volume of liquid scintillator is $1120 \mathrm{~cm}$.

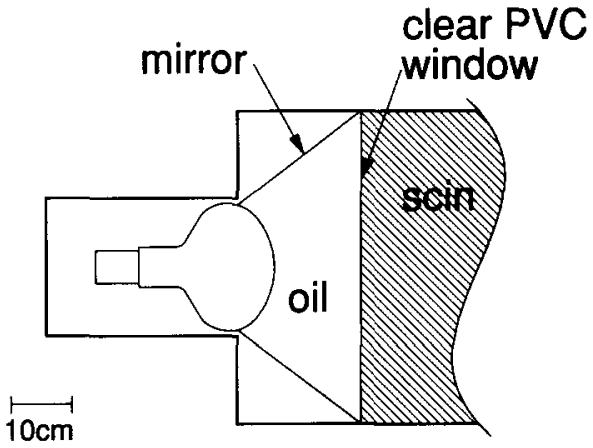

Fig. 4. Details of each end of the vertical scintillation counters. The length of the sensitive volume of lıquid scintıllator is $1107 \mathrm{~cm}$.

\subsection{The scintillator counters}

Both the horizontal and vertical counters are of uniform rectangular cross section and consist of three chambers separated by transparent PVC windows. The middle chamber is the major one and is filled with liquid scintillator. The two end chambers contain both the light collection optics and the photomultipliers. They are filled with pure mineral oil of the same type as used for the base of the scintillator. This geometric arrangement provides ideal optical contact between the scintillator and the PMs and limits the dynamic range of PM signals caused by radioactive decays near the photomultiplier itself. Furthermore, it allows reasonably convenient maintenance access to the photomultipliers. The detailed geometry of these end chambers is shown in fig. 3 for the horizontal scintillators and in fig. 4 for the vertical ones.

The length of the scintillator chamber of the horizontal counters is $1120 \mathrm{~cm}$ and its width is $73.2 \mathrm{~cm}$. The nominal depth of scintillator oil is $19 \mathrm{~cm}$, but this varies by a few millimeters from tank to tank. Similarly, since the tanks are not perfectly level, there is a few millimeters depth difference between the two ends, also varying from tank to tank. The inside walls of the scintillator tanks are lined with a commercial white vinyl-FEP material \#2. Since the FEP has a refractive index of 1.33 and the mineral oil/scintillator mix has an index of $1.4750 \pm 0.0005$, there is total internal reflection from the walls for light inside a critical angle of $\sim 25.6^{\circ}$. The air/scintillator interface for the top surface also provides total internal reflection. Tests made on scale model tanks with small transverse di-

\#2 This material is manufactured by Norton Chemplast, Wayne, N.J., under the trade name "Chemflour". It is a white vinyl material with an acrylic pressure sensitive adhesive on one side and a 0.001 in. film of FEP on the other. 
mensions indicate that the effective attenuation length due to reflections on the liner is greater than $26 \mathrm{~m}$. Since the observed attenuation length of the counters is somewhat less than this, the liner is not a limiting factor in the performance of the counters. Finally, there is no evidence of any chemical or physical interactions between the liquid scintillator and this liner material over a period of several years.

In order to provide the possibility of conveniently monitoring the scintillator response with an artificial light source, a slotted PVC pipe is run from one end of the counter to the other. This pipe is mounted on the inside top surface of the scintillator tank and is transversely centered. Quartz optical fibers guiding UV laser light to various points inside the counter are passed through the slotted pipe. The calibration light sources are described in more detail below.

The end chambers are separated from the scintillator by a window made of $0.32 \mathrm{~cm}$ clear PVC ${ }^{\# 3}$. This material has an optical transmission of $>90 \%$ for wavelengths longer than $400 \mathrm{~nm}$; it cuts off sharply at about $390 \mathrm{~nm}$. For the horizontal counters, the end chambers contain two light collection mirrors with one LED mounted on each, two PMs, closed cell polyfoam PM mounts to support the buoyant forces on the PM, and the PM bases and their cabling. They are filled with pure mineral oil to the same depth as the scintillator.

In the horizontal counters the two $20 \mathrm{~cm}$ diameter photomultipliers (Hamamatsu R-1408) cover only $47 \%$ of the transverse area of the counter. Therefore, for light produced at points in most of the volume of the counter, unaided PMs intercept about half of the light that reaches the end chamber. Since the photomultipliers have an hemispherical photocathode, we were able to design short mirrors with good geometric light collection efficiency by allowing the PM envelope to be re-entrant into the mirror. The mirror shape is a surface of revolution, suitably truncated to fit into the rectangular cross section of the counter. The shape of the revolved planar curve was calculated so that a ray coplanar with the mirror axis and making an angle of $31^{\circ}$ with it was reflected tangent to the surface of the re-entrant PM. This condition guarantees that all such coplanar rays that can be transmitted from a distant source are reflected onto the phototube envelope. Measurement of the response of the photocathode as a function of the angle which incident light makes with the envelope normal, $\theta$, shows a $\sec \theta$ response up to $60^{\circ}$ and an almost constant response from $60^{\circ}$ to near tangency. Thus, the design is optimal for light coplanar with the axis of revolution. Skew rays can be lost, but

\footnotetext{
*3. This material is manufactured by Robeco Chemicals, Inc.,
} under the name "A-100 clear PVC".
Monte Carlo calculation shows that for light within the bundle transmitted from a distant source, the mirror geometric efficiency is greater than $99 \%$.

The mirrors are manufactured by vacuum forming acrylic plastic over a precisely cut aluminum form. These mirror blanks are then front surface aluminized. Measurements of a counter's response at one end to muons passing through the counter near the other end show that the light collecting mirrors increase the signal size a factor of 1.71 . This is to be compared with an ideal increase of 2.13 for perfect light collection, so that the overall light collection efficiency of the mirrors is $80 \%$. The mirrors leave unchanged the response of one end to muons passing near that end. Thus on average, the mirrors increase signal size and decrease dynamic range.

In general, the geometry of the vertical counters is similar to that of the horizontal ones. The length of active scintillator is $1107 \mathrm{~cm}$ and it is $21.7 \mathrm{~cm}$ wide. The oil depth is $46.2 \mathrm{~cm}$, varying from tank-to-tank and end-to-end by a few millimeters. The walls are lined with the same material as for the horizontal tanks. The gcometry of the end chambers is shown in fig. 4 above. Only one phototube is used at each end and the light collection mirror is a simple cone truncated suitably to fit into the rectangular cross section of the counter. The cone is developed from flat sheets of highly reflective aluminum ${ }^{\# 4}$.

\subsection{The liquid scintillator}

Because of the extraordinary length of the MACRO scintillation counters, the critical parameter for the scintillator oil is its transparency. Ideally, its attenuation length at the wavelength of the scintillation light should be at least $12 \mathrm{~m}$. This has been achieved by using a high purity mineral oil base and choosing the amount of scintillant to maximize the signal from the far end of a counter. The mineral oil base has an attenuation length of $20 \mathrm{~m}$ or more at a wavelength of $425 \mathrm{~nm}$ [2]. Fig. 5 shows measurements made on a long path laser spectrophotometer of a typical sample of the base oil used in MACRO. The scintillant is a mixture of: pseudocumene (1,2,4-trimethylbenzene); PPO (2,5diphenyl-oxazole); and bis-MSB (p-bis[o-methylstyryl] benzene). The absorption and fluorescence spectra of these materials are given in ref. [3].

Preliminary measurements in a $12 \mathrm{~m} \times 25 \mathrm{~cm} \times 25$ $\mathrm{cm}$ test tank were used to set the relative concentrations to $40 \mathrm{~g}$ PPO and $40 \mathrm{mg}$ bis-MSB to one liter of pseudocumene $(876 \mathrm{~g})$. Furthermore, these measurements suggested that the concentration of pseudo-

\#4 This material is manufactured by Alcoa Aluminum under the trade name "Everbrite". A lower limit on its spectral reflectivity is $85 \%$. 


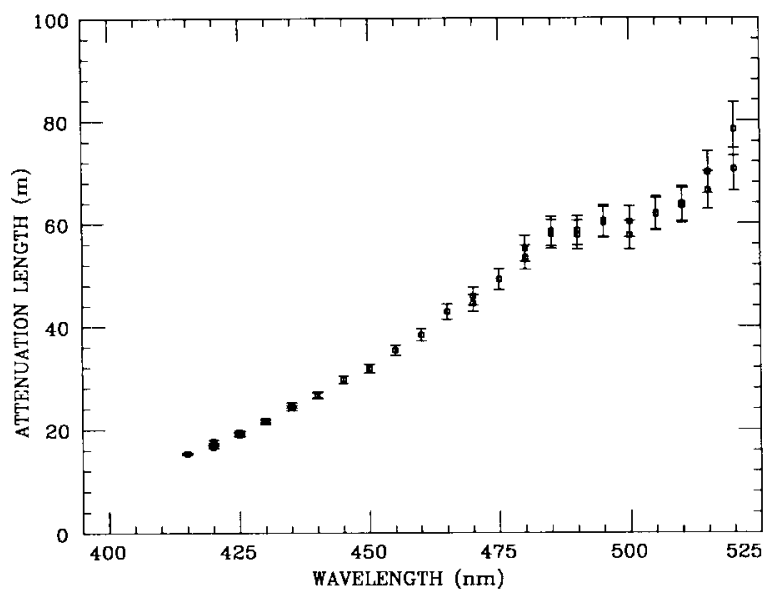

Fig. 5. Long path laser spectrophotometer measurements on a typical sample of MACRO base oil (Britol 6NF oil).

cumene mixture in mineral oil should be $6.2 \%$. Twenty-nine of the counters were filled with this mixture in June, 1988. Further work was done using a full sized horizontal counter. Its response function was measured as the amount of scintillant was gradually increased. Fig. 6 shows the light yield from a muon passing through the far end of a counter as a function of the percentage concentration of the pseudocumene mixture in the mineral oil. On the basis of these measurements, it was decided to use a conservative pseudocumene concentration of $3.6 \%$. The remaining twenty-four counters in supermodule 1 were filled with this concentration and it will be used for the remainder of the MACRO detector. The final MACRO scintillator is then: $96.4 \%$ mineral oil, $3.6 \%$ pseudocumene, $1.44 \mathrm{~g} / 1$ of PPO, and $1.44 \mathrm{mg} / 1$ of bis-MSB. In addi-

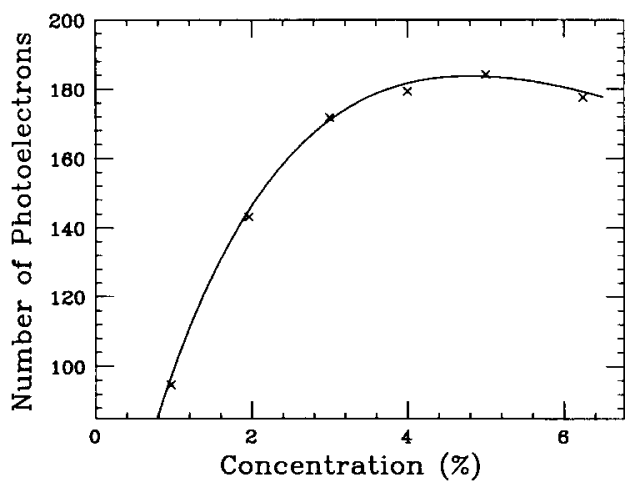

Fig. 6. Light yield vs pseudocumene concentration for a muon passing through the far end of a MACRO test counter. The final scintillator mixture with improved light attenuation yielded about a factor of 2 more photoelectrons than indicated here. tion, the mineral oil supplied by the manufacturer contains $40 \mathrm{mg} / \mathrm{l}$ of an antioxidant. After each batch of scintillator is mixed, the final concentrations of the active ingredients are checked by quantitative spectrophotometry before the counters are filled.

\subsection{The scintillator calibration system}

For purposes of setting up the electronics and monitoring scintillator system stability, artificial light sources have been installed in each counter. Available are LEDs mounted near the PM in the counter end chambers and UV light from a nitrogen laser coupled to the liquid scintillator by optical fibers.

The LEDs are mounted on the mirrors in each horizontal counter and on the clear window in the vertical counters. They allow individual excitation of the counters with light pulses which simulate monopoles, muons, and fractionally charged particles, and also provide a convenient source of very small light pulses to excite single photoelectron signals. We chose the HLMP-3001 red LED manufactured by HewlettPackard, since out of all the LEDs we tested, it has the least overshoot for microsecond length pulses which are necessary for simulating monopoles. It has a rated "speed of response" of $10 \mathrm{~ns}$. We find that for long pulses, it has a $60 \mathrm{~ns}$ to $80 \mathrm{~ns}$ rise time. Furthermore, it is sufficiently bright with modest drive to simulate a monopole of any velocity. The laser calibration system is based on a type VSL337 nitrogen laser \#5. This instrument produces a 3 ns FWHM pulse of $337.1 \mathrm{~nm}$ UV light. This wavelength is well below the absorption band of pseudocumene, just on the high edge of that of PPO, and at the peak absorbency of bis-MSB. Thus, the light seen by the phototubes has essentially the same spectral and temporal distributions as that produced by normal ionization events. The laser output is passed through a variable attenuator and then into one of two splitters, each driving one quartz fiber per scintillation counter. The fibers from one splitter terminate at the longitudinal center of each counter and those from the other terminate $2 \mathrm{~m}$ from one end. The splitter is manually determined and usually the one driving the centered fibers is used. Thus, a single laser pulse illuminates all the counters. In addition, one fiber directly from the laser and another on the output of the attenuator drive two special monitoring photomultipliers. The one directly from the laser provides us with a normalizer to eliminate the $\pm 5 \%$ pulse-to-pulse variations of the laser and that which passes through the attenuator provides a cross check on it. At four

\#5 Model 33700 VSL-337 nitrogen laser, manufactured by Laser Science, Inc., Cambridge, MA. $40 \mathrm{KW}$ peak power at $337 \mathrm{~nm}, 3 \mathrm{~ns}$ FWHM, $100 \mu \mathrm{J}$, repetition rate $1-20 \mathrm{~Hz}$. 
hour intervals during detector operation, both the LEDs and the laser are fired about 100 times using a fixed set of excitation parameters. This allows rapid detection of system malfunctions and the creation of a data base from which to assess counter stability. In addition, once per week the system is exercised much more extensively with a wide pattern of excitation parameters allowing exhaustive checks and calibrations of the electronics.

\subsection{Performance of scintillation counters}

From the ratio of the refractive index of the FEP to that of the oil and the transverse dimensions of the counters, it can be shown that $\approx 6 \%$ of the light produced at a point farther than about $1 \mathrm{~m}$ from a counter end is geometrically accepted by that end. Measurements comparing the response of samples of the MACRO scintillator (3.6\% pseudocumene concentration) to that of a geometrically similar sample of plastic scintillator show that its absolute efficiency is $0.94 \%$. (The light generation efficiency of the " $6 \%$ pseudocumene scintillator" is $1.15 \%$.) The calculated average energy loss of a minimum ionizing particle passing through $19 \mathrm{~cm}$ of this scintillator is $34 \mathrm{MeV}$ and the most probable energy loss is $31 \mathrm{MeV}$. Thus, ignoring light attenuation of the scintillator, on average 4900 photons $(3 \mathrm{eV})$ reach photocathodes when a muon passes normally through the scintillator.

Measurements of the response of a horizontal tank to penetrating muons were made by defining beams of cosmic ray muons with small plastic scintillator telescopes at eight positions along the counter. Fig. 7 shows a worst case counter response curve; in general, the scintillator used in MACRO has less attenuation than that used for these measurements. The calibration of the PM response from signal size to "conventional photoelectrons" was done using the mean and the rms width of its response to LED light flashes. It

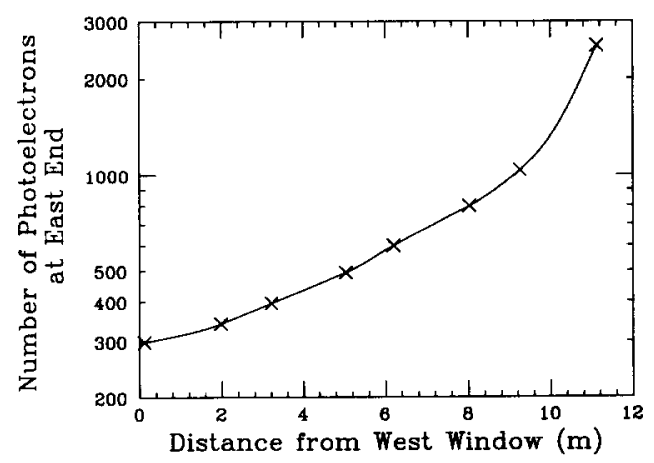

Fig. 7. Scintıllation counter response curve showing the light yield vs muon position in the counter. does not take into account the contribution to the width of the distribution from the statistical gain of the PM. The ratio $300 / 4900=6.1 \%$ of the number of conventional photoelectrons detected from the far end of the counter to the expected amount of light successfully transmitted toward the PM includes the attenuation of the fight in the scintillator, the photocathode efficiency, and the effect of the PM gain statistics.

\subsection{The scintllator electronic instrumentation}

In the sections that follow, we give an overview of each electronic system in use for the MACRO scintillation counters. Many of the circuits have been custom designed and built by various collaborating institutions. Detailed technical articles have been published on specific systems; hence, we refer the reader to the cited references for more comprehensive descriptions.

\subsubsection{The photomultiplier tubes and front-end electronics}

The photomultipliers used in the first supermodule are Hamamatsu R-1408. These PMs have $20 \mathrm{~cm}$ diameter hemispherical photocathodes and $13 \mathrm{CeS}$ dynodes in a fast venetian blind structure. Each anode of the two tubes in one horizontal counter end drives a short $93 \Omega$ cable paralleled by a $92 \Omega$ terminating resistor; the two $93 \Omega$ cables are simply connected to one $50 \Omega$ cable that goes to the electronics. Pulses from the single photomultiplier in the vertical counter ends are brought out directly on a $50 \Omega$ cable. The PMs are operated at a nominal charge gain of $1 \times 10^{7}$ to yield an average peak pulse height from a single photoelectron at the input to the electronics of $3 \mathrm{mV}$. The typical high voltage is about $-1500 \mathrm{~V}$; this voltage is divided so that there is about $550 \mathrm{~V}$ between the cathode and the first dynode and the other $950 \mathrm{~V}$ is uniformly divided among the dynodes. At this gain the typical rate of dark noise pulses above the mean single photoelectron level is below $1 \mathrm{kHz}$, the rms deviation of the transit time from its mean for full cathode illumination of 100 photoelectrons or more is less than $1.5 \mathrm{~ns}$, and the rate of afterpulses above the single photoelectron level in a time window from $0.2 \mu \mathrm{s}$ to 10 $\mu s$ is less than $1 \%$ for each photoelectron. The distribution of output charge from single photoelectrons is monotonically decreasing with no hint of either a peak or even a shoulder; it is well approximated by a simple exponential. The FWHM of the output pulses from a single photoelectron is $8 \mathrm{~ns}$.

Finally, the anode output charge as a function of the number of photoelectrons for a small signal gain of $5 \times 10^{6}$ is shown in fig. 8 . The dynode saturation characteristic is similar to that of the anodes, but not quite as severe; the dynamic range of the system is improved by a factor of about 5 by use of the dynode output. 


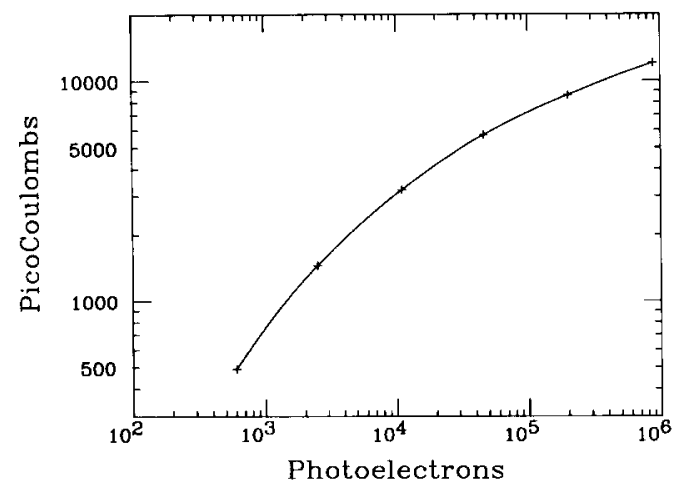

Fig. 8. Photomultiplier output charge as a function of the number of input photoelectrons for a PM gain $=5 \times 10^{6}$. The smooth curve through the data points serves as a guide to the eye only.

In a study of vertical muons through the two scintillator planes, the sigma of the measured time resolution of a single counter is of order 1 ns indicating MACRO's ability to distinguish downward from upward going particles.

\subsubsection{Monopole triggering and waveform recording}

A slowly moving magnetic monopole traversing a MACRO scintillation counter may produce very little light. The signal may be a wide pulse of low amplitude or may breakup into a train of single photoelectron pulses lasting for several microseconds. Hence, the central problem to be addressed by the "slow monopole trigger" is to be sensitive to various signals, from wide pulses of low amplitude to trains of single photoelectron pulses.

The slow monopole trigger circuit used in MACRO has two parts. The first is an analog circuit that provides a TTL output pulse whose duration is nominally the time that the input pulse is larger than its half height. Thus, it is called the time-over-half-max (TOHM) circuit. There is a minimum pulse size required at the input for the circuit to operate and this is set to below the size of the average single photoelectron pulse height. These circuits are driven by an amplified anode signal $(\times 10)$ from the PM signal fanouts. By taking the width at half maximum of the pulses and disregarding the pulse heights, the TOHM circuits effectively suppress large and sharp pulses produced by muons and radioactive decays. In this manner a sharp pulse of 200 photoelectrons, for example, produces a TOHM output similar to a single photoelectron pulse, while a train of 20 single photoelectron pulses produces 20 such TOHM outputs. In supermodule 1 , the scintillators were grouped into units of four for driving the TOHMs. The typical output rate of each group was about $120 \mathrm{kHz}$. In the final configuration of MACRO, each scintillator end will have its own TOHM circuit.

The second part of the slow monopole trigger circuit uses the TOHM output as input. This digital circuit counts a scaler up at a fixed high frequency (66 $\mathrm{MHz}$ ) while the input signal from the TOHM is high and counts down at a relatively low frequency while the input signal is low. The circuit triggers if the count in the scaler exceeds a preset limit. Because the scaler count rises linearly while there is input and falls linearly at a slower rate when no input is present, the circuit is called a leaky integrator (LI). It is easy to see that for suitably adjusted values of its two parameters (the down counting frequency and the preset count for triggering), this arrangement will reject narrow pulses due to muons and detect both wide pulses and sufficiently dense bursts of single photoelectrons. The LI circuit uses standard TTL digital logic and each TOHM circuit drives one LI. A coincidence within $20 \mu$ s of LI outputs from the two ends of a group of scintillators is used to trigger the data acquisition and to stop the waveform digitizers at an appropriate time so that the details of the triggering waveform can be studied. At the time each waveform digitizer is stopped, a time word from a $5 \mathrm{MHz}$ clock is latched. This time word records the relative timing of the waveform data from different channels and can be used to calculate the time-of-flight of a slow monopole.

Two waveform digitizers were used in conjunction with the slow monopole trigger system. A LeCroy 2262 system, clocked at $50 \mathrm{MHz}$, covers a $6 \mu \mathrm{s}$ window centered on the triggering event. A slower, custom built system is clocked at $20 \mathrm{MHz}$ and covered a $20 \mu \mathrm{s}$ window [5]. The signals into the $50 \mathrm{MHz}$ system are attenuated by a factor of 5 while the signals into the 20 $\mathrm{MHz}$ system are amplified by a factor of 10 so that the two systems cover different dynamic ranges.

The triggering efficiency of the slow monopole trigger system as a function of total signal charge for a fixed duration of input signal falls very steeply from unity to zero near a triggering threshold. The input charge corresponding to a $90 \%$ triggering efficiency for each pulse duration is mapped by exciting the scintillator PMs with LED pulses. Fig. 9 shows the measured slow monopole trigger sensitivity as a function of particle velocity for the horizontal counters with trigger parameters used for MACRO data taking in 1990. In addition, fig. 9 shows the expected light yield for various types of particles [6].

In addition to slow monopole detection, MACRO is instrumented to detect the passage of relatively fast monopoles with velocities in the range $2.5 \times 10^{-3}<\beta$ $<1.5 \times 10^{-2}$. This trigger is generated by the fast particle triggers (described below) with the addition of a veto of prompt muon events. This trigger overlaps 


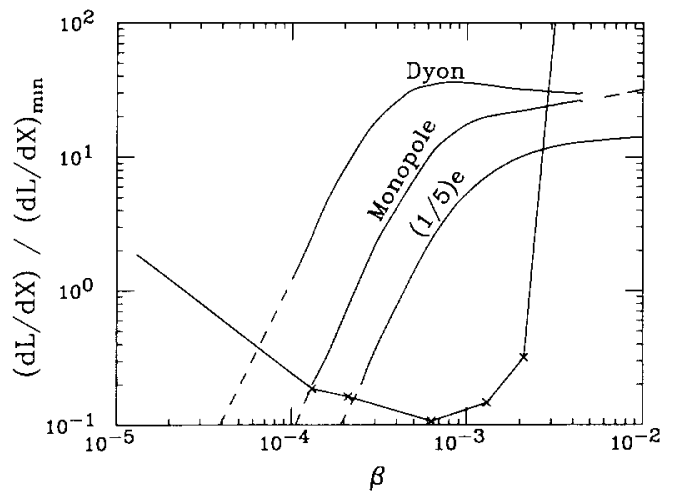

Fig. 9. The curve with data points $(x)$ shows the monopole trigger sensitivity versus particle velocity. The circuit triggers with $90 \%$ probability when the amount of light equals the value given by the sensitivity curve. The curve serves as a guide to the eye only. The expected light yield for various particle types [6] is indicated by the labeled curves.

the velocity range of the slow monopole trigger. In all cases, the scintillator waveforms are recorded.

\subsubsection{Fast particle triggering and inital $A D C / T D C$ electronics}

The fast particle trigger in MACRO treats each detector face as a single large scintillation counter. The PM signals from each side of all the scintillators on a face of the detector are linearly added together and discriminated at a low enough level to be $100 \%$ efficient for muons but high enough to reject essentially all the radioactive background signals. The discriminated signals from the two sides of a face are put in $100 \mathrm{~ns}$ coincidence (the light transit time through a scintillator is about $60 \mathrm{~ns}$ ), and finally each pair of these face signals is put into a $1 \mu$ s coincidence to form the fast particle trigger.

Because of its availability at the time of turn on of MACRO's first supermodule, electronics used by the IMB proton decay experiment [7] was modified for use as a triggering and $\mathrm{ADC} / \mathrm{TDC}$ system for the scintillation counters. In this electronics, discriminated signals are derived from the input circuitry of the ADCs and TDCs and are used to produce a trigger for particles passing through any two faces of the MACRO detector with transit time less than $7 \mu \mathrm{s}$ This corresponds to a velocity of $\beta>2.5 \times 10^{-3}$ for a particle passing vertically through the apparatus. The discrimination level on each scintillator's end is set at about the 30 photoelectron level. This level is well below the single muon signal and yet results in an acceptably low trigger rate due to accidentals from the radioactivity background. Coincidences within 100 ns are set up between the two ends of each scintillator and these are ORed over all the scintillators in a face to generate a pre-trigger. A fast particle (muon) trigger is generated by a $7 \mu \mathrm{s}$ coincidence between any pair of these pre-triggers. When triggered, the system measures both the relative time of occurrence and the charge in both the anode and dynode signals from all the scintillators. The IMB electronics served as the primary muon trigger and ADC/TDC system for the first supermodule of MACRO. However, since time of turn-on, more sophisticated systems have been developed which will be used for all of MACRO. These systems are described in the following two sections.

\subsubsection{The energy reconstruction processor}

The energy reconstruction processor (ERP) system (fig. 10) is an integrated trigger and ADC/TDC read-
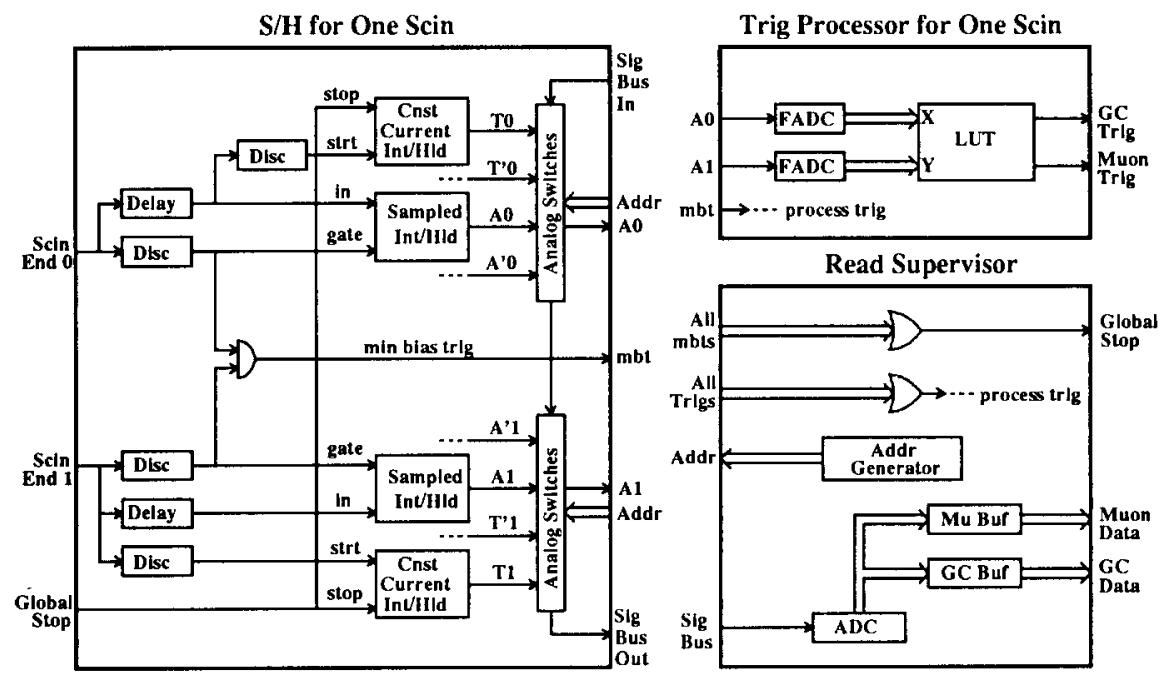

Fig. 10. General logic of the energy reconstruction processor (ERP) used by the MACRO detector. 
out system for the MACRO scintillator counters. It provides a general muon trigger that does not require coincidence between counters, precision ADC and TDC digitizations of scintillator signals, and a gravitational stellar collapse trigger. The system is composed of three separate module types: the sample-and-hold module $(\mathrm{S} / \mathrm{H})$, the trigger processor module, and the readout supervisor module.

The $\mathrm{S} / \mathrm{H}$ module forms the front end of the system. Its primary function is to hold the PM signal amplitude and timing information for either readout or triggering purposes. For amplitude information, it integrates the PM signals and, for timing information, it integrates constant current pulses whose lengths measure the relative occurrence times of the PM signals. It holds this information as analog voltages and makes them available to a digital triggering circuit in the trigger processor and, when a trigger is generated, to a precision digitizer in the readout supervisor. In addition, the $\mathrm{S} / \mathrm{H}$ circuitry includes discriminators on the input signals, logic to generate a minimum bias trigger for internal use, ADC charge integration gating logic, and output multiplexing used during readout.

The discriminators, whose thresholds are CAMAC programmable, produce $200 \mathrm{~ns}$ long pulses, which form the charge integration gates. This width is appropriate both for integrating the $50 \mathrm{~ns}$ wide PM signals and for accommodating the maximum light transit time of 60 ns from one end of a counter to the other. To enable this integration gate to start before the PM signal arrives, the PM signals to be integrated are passed through 140 ns delay lines on the $\mathrm{S} / \mathrm{H}$ module.

Each delayed PM signal is split into a direct output (the $\mathrm{A} 0$ and $\mathrm{A} 1$ in fig. 10) and one attenuated by a factor of $10\left(\mathrm{~A}^{\prime} 0\right.$ and $\mathrm{A}^{\prime} 1$ in fig. 10). The attenuated version of the signal allows the entire dynamic range of the PM to be on-scale in the ADC circuitry. Each of these signals drives a voltage-to-current amplifier whose output is fed through a fast analog switch to an integrating and hold capacitor. Thus amplitude data for a single counter consists of four ADC values.

Separate discriminators (giving time signals $\mathrm{T}$ and $T^{\prime}$ ), identical to those used in the trigger generation, but with independent thresholds, turn on a constant current into the integrating capacitors. This current is turned off by a global stop signal formed by the delayed logical OR of all minimum bias triggers in the system. Thus the voltages on the capacitors provide relative timing information on all PM signals involved in an event. The use of two independent timing thresholds provides redundancy in the timing measurement, helping to eliminate the effects of PM pre-pulsing. Hence, timing data for a counter also consists of four values.

An overlap of the discriminator outputs from both ends of a counter produces the minimum bias trigger, which is the requirement for subsequent processing of an event. In the absence of a minimum bias trigger, the $\mathrm{S} / \mathrm{H}$ values are reset within $200 \mathrm{~ns}$ and the event rejected without participation of the trigger module. If a minimum bias trigger is obtained, the integrated signals are held and a trigger module processing cycle is initiated.

After a $1 \mu \mathrm{s}$ delay to allow the $\mathrm{S} / \mathrm{H}$ voltages to settle, the trigger processor performs rapid 6-bit digitizations of the $\mathrm{S} / \mathrm{H}$ analog information for both ends of a counter. The two amplitude digitizers have a nonlinear (hyperbolic) response given by $d_{\text {out }}=V_{\mathrm{in}} /\left(B V_{\mathrm{in}}+\right.$ $V_{0}$ ), where $B$ (amount of nonlinearity) and $V_{0}$ are constants. These digitizations are used as addresses (X and $\mathrm{Y}$ ) into an $8 \mathrm{k} \times 8 \mathrm{k}$ RAM look-up table (LUT). These tables are pre-loaded with suitable information to produce two types of triggers: one occurs for every PM signal combination that a muon can produce in a counter (equivalent to an energy threshold of about 10 $\mathrm{MeV}$ ) and the other, for every combination associated with an energy of about $5 \mathrm{MeV}$.

When a trigger occurs, the supervisor controls the readout of the system. The key element of the supervisor is a 12 bit, $1 \mathrm{MHz} \mathrm{ADC}$, into which the $\mathrm{S} / \mathrm{H}$ voltages are fed sequentially. The digitized amplitude and timing words are stored in two buffers. The first of these is loaded only for the case of a muon-type trigger and is presented for immediate CAMAC readout. The second buffer is circular and contains a history of the previous $\sim 800$ events of both trigger types. This buffer is readout at the time of its wrap-around, approximately every $10 \mathrm{~min}$ for the settings used in the first supermodule run. The TDC information readout by the ERP system has a least count of $1 / 6$ ns and the $\mathrm{ADC}$ has a least count of $1 \mathrm{pC}$.

The $\mathrm{S} / \mathrm{H}$ module is packaged as a $9 \mathrm{U}$ VME module and contains circuitry for four counters. The PM inputs are provided to the module through a ribbon coaxial cable. The ERP trigger processor module is packaged as a single width CAMAC unit which services four scintillator counters. The readout supervisor is also a single width CAMAC unit and services the $\mathrm{S} / \mathrm{H}$ and trigger processor modules for one supermodule.

\subsubsection{Pulse height recorder and synchronous encoder}

The pulse height recorder and synchronous encoder (PHRASE) circuit (fig. 11) was developed for MACRO as a specialized trigger and recording system for gravitational stellar collapse events. It provides a waveform recording capability complementary to the ones triggered by the TOHM and other circuits. To detect stellar collapse events, the large radioactivity background must be rejected in hardware. Due to light attenuation, a certain energy released in a scintillation counter may correspond to PM pulse amplitudes differing by a factor up to three. Hence, radioactivity 
1/2 PHRASE Unit

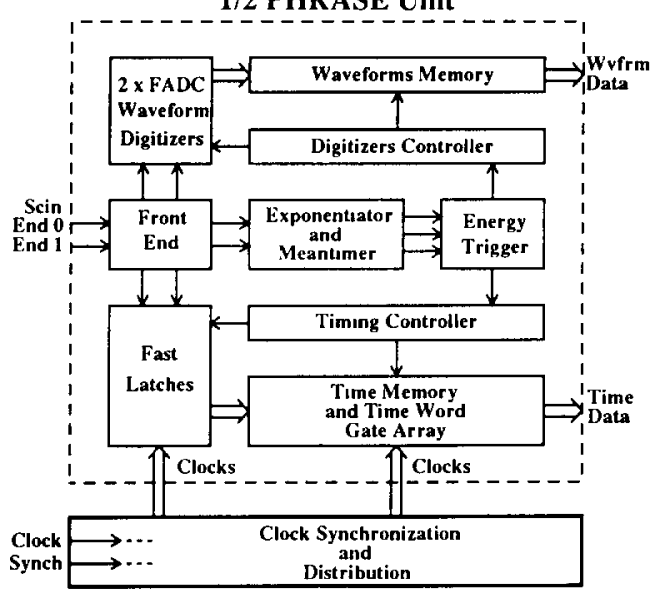

Fig. 11. General logic of the pulse height recorder and synchronous encoder (PHRASE) used by the MACRO detector.

rejection by energy threshold depends on fast hardware energy reconstruction, independent of the event position within the counter. A trigger circuit for gravitational stellar collapse detection must therefore provide such a primary energy threshold in the range $E=5-10 \mathrm{MeV}$. Moreover, if one wishes to detect the $2.2 \mathrm{MeV}$ photon that may result from n-capture in hydrogen, the energy threshold must be lowered to $\sim 1 \mathrm{MeV}$ for a time at least comparable to the n-capture time in scintillator $(\sim 180 \mu \mathrm{s})$ after detection of a primary event.

The PHRASE electronic modules are built in a CAMAC standard. Each module serves two scintillation counters. The main circuit characteristics are:

1) It provides (in $\approx 80 \mathrm{~ns}$ ) a trigger signal ET (energy trigger) when a threshold condition is satisfied on the energy released by an event in a MACRO counter. The primary energy threshold $\left(E_{\mathrm{pth}}\right)$ is independent from the event position within the counter.

2) It digitizes waveforms of the signals from both counter ends and stores them with automatic zero suppression only when an ET has occurred.

3) It measures with an accuracy $\sim 1.6 \mathrm{~ns}$ the time of each event relative to a UTC clock.

4) Each time an ET is obtained from one counter, the PHRASE thresholds for both counters read by the module are lowered (in $\sim 80 \mathrm{ns)}$ ) to a different programmable value $\left(E_{\mathrm{sth}}\right)$ for a pre-settable time interval ST (special time). A maximum of 14 events can be stored during the ST.

5) A CAMAC LAM is generated at the end of the ST to trigger the data acquisition.

The energy trigger works according to the following principles. If $L$ is the MACRO counter length and one chooses a longitudinal coordinate axis $x$ with origin at the counter center, the two counter ends are identified by the $x$-values $x=-L / 2$ and $x=+L / 2$ for the "left" and "right" ends respectively. Since light attenuation in the counter is exponential to very good approximation (apart from the last meter close to the PMs), if one calls $\lambda$ the attenuation length $(\lambda \sim 12 \mathrm{~m})$, the pulse amplitudes for the "left" and "right" PMs can be written:

$A_{\mathrm{L}}(E, x)=\alpha E \mathrm{e}^{-x / \lambda} ; \quad A_{\mathrm{R}}(E, x)=\alpha E \mathrm{e}^{+x / \lambda}$,

for an event releasing visible energy $E$ at the longitudinal position $x$ inside the counter. The light arrival time at the two PMs can be written:

$t_{\mathrm{L}}=t_{0}+x / v ; \quad t_{\mathrm{R}}=t_{0}-x / v ; \quad v \approx 0.2[\mathrm{~m} / \mathrm{ns}]$.

Now, if an exponential time-tail is electronically added to the peak amplitude signals, these can be written:

$S_{\mathrm{L}}(E, x, t) \propto E \mathrm{e}^{-x / \lambda} \mathrm{e}^{-\left(t-t_{\mathrm{L}}\right) / \tau} ;$
$S_{\mathrm{R}}(E, x, t) \propto E \mathrm{e}^{+x / \lambda} \mathrm{e}^{-\left(t-t_{\mathrm{R}}\right) / \tau}$,

for $t \geq t_{\mathrm{L}, \mathrm{R}}$; otherwise $S_{\mathrm{L}}$ and $S_{\mathrm{R}}$ are 0 .

If one introduces the "mean time" $t_{\mathrm{MT}}=\left(t_{\mathrm{L}}+t_{\mathrm{R}}\right) / 2$ and if $\tau$ is chosen such as $\tau=\lambda / v$, the previous formulae simplify to:

$S_{\mathrm{L}}(E, x, t) \propto E \mathrm{e}^{-\left(t-t_{\mathrm{MT}}\right) / \tau} ;$

$S_{\mathrm{R}}(E, x, t) \propto E \mathrm{e}^{-\left(t-t_{\mathrm{MT}}\right) / \tau}$,

for $t>\operatorname{MAX}\left(t_{\mathrm{L}}, t_{\mathrm{R}}\right)$.

This means that, for times greater than the maximum between $t_{\mathrm{L}}$ and $t_{\mathrm{R}}$, the exponential tails are overlapping and their amplitudes, at a fixed delay $t^{*}$ after $t_{\mathrm{MT}}$, are only functions of energy; the exponential nonuniformity has been canceled. In MACRO, $\tau \approx 60$ ns and we chose $t^{*}=40 \mathrm{~ns}$, which always satisfies the condition $t>\operatorname{MAX}\left(t_{\mathrm{L}}, t_{\mathrm{R}}\right)$. The exponential tails are continuously compared with the primary energy threshold and the discriminator outputs go to a three-fold coincidence together with a $t_{\mathrm{MT}}$ signal. The coincidence requires that both the L-tail and the R-tail be above threshold when the $t_{\mathrm{MT}}$ signal arrives; an "energy trigger" ET is then generated.

The PHRASE circuit provides a waveform digitization that is based on techniques developed for the circuit GATHER by Francois Bourgeois at CERN [8]. The two PM signals are continuously sampled by nonlinear (hyperbolic compression) waveform digitizers, based on 6-bits, $100 \mathrm{MHz}$ Flash ADCs. The 8-bit equivalent dynamic range is 1 to $256 \mathrm{mV}$.

The $100 \mathrm{MHz}$ clock, externally distributed to all the PHRASE circuits, is also used to measure the light arrival time at both counter ends. Three continuously running time counters are driven by the clock. The first counter is a simple $12.5 \mathrm{MHz}, 24$-bit binary counter; its time depth is therefore $\sim 1.3 \mathrm{~s}$; its $80 \mathrm{~ns}$ period is further subdivided into $1.6 \mathrm{~ns}$ intervals by two other clocks. One of these is a four-line Gray code counter 


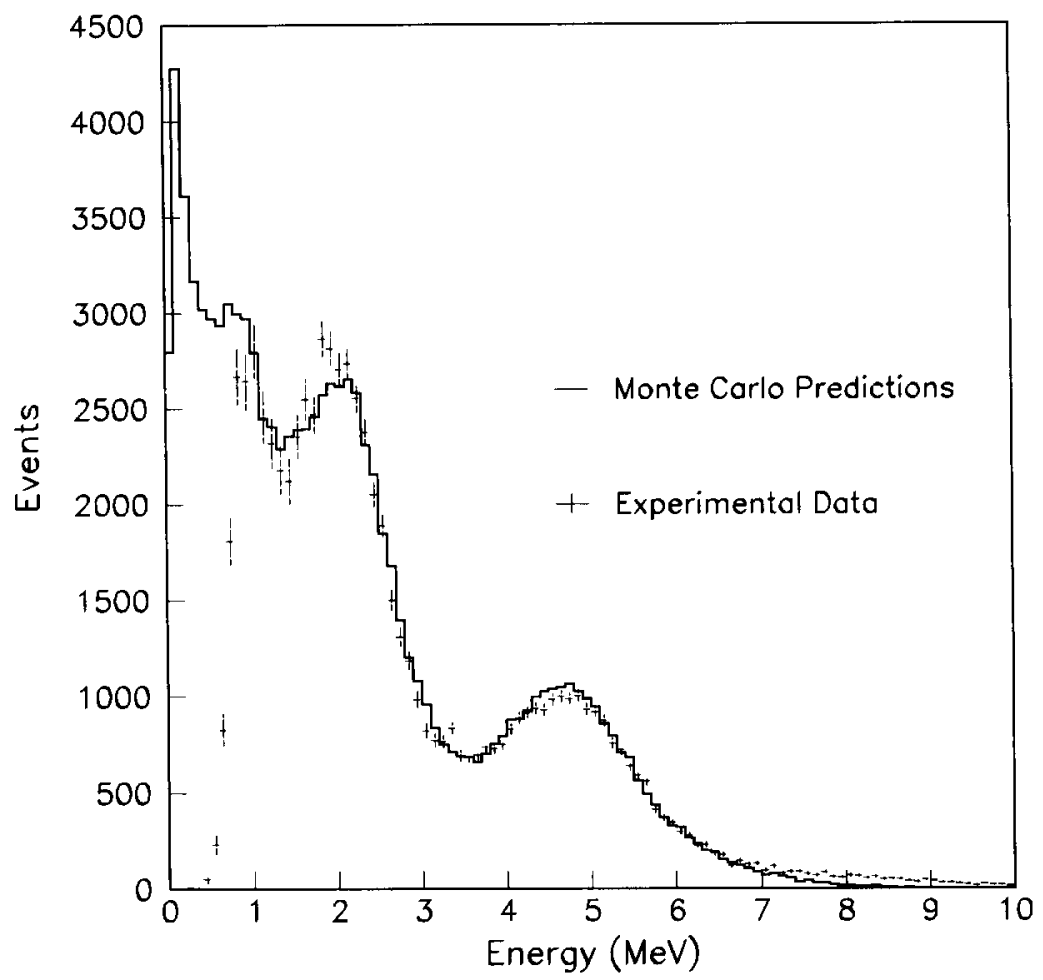

Fig. 12. The energy spectrum for events induced by the Am/Be source. The 4.44 and the $2.2 \mathrm{MeV}$ gamma-lines are visible. The full-line histogram is the Monte Carlo simulation.

(10 ns period, 160 ns time-depth); the other has a period of $1.6 \mathrm{~ns}$ obtained by splitting the $100 \mathrm{MHz}$ clock into three lines that are relatively delayed by 1.6 ns (Johnson code). The Gray and Johnson codes change only one bit at a time thus allowing an asynchronous latching of their status when signals from the scintillation counter pass specific thresholds. The time information of the three time counters is stored in a GATE

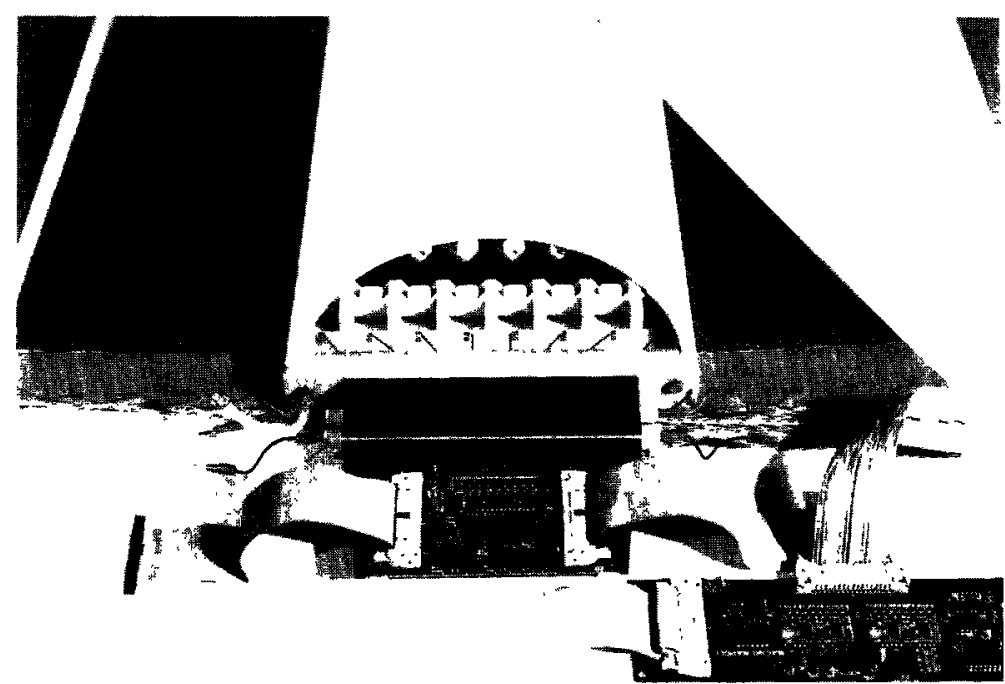

Fig. 13. Details of an 8 cell streamer tube chamber of MACRO. The PVC envelop has been cut in order to show the inner part of the chamber. The wire and strip readout cards are also shown. 
ARRAY chip, custom made for PHRASE. This method of time measurement does not depend on any calibration; it allows a time resolution better than 2 ns between the signals from the two counter ends or between any two events from different scintillation counters.

The PHRASE electronics has been used to determine an absolute calibration of a typical MACRO scintillation counter at low energy. An Am/Be source (external to a scintillation counter) which emits neutrons as well as a $4.44 \mathrm{MeV}$ gamma-line was used. Since a large fraction of the emitted neutrons $(\approx 60 \%)$ are followed by a cascade $4.44 \mathrm{MeV}$ gamma-emission, one can also perform more refined tests [9]. Delaycd $\mathrm{n}$-capture, tagged by the $4.44 \mathrm{MeV}$ gamma-line, after neutron moderation and diffusion in the liquid scintillator can be studied. The $2.2 \mathrm{MeV}$ gamma-rays, produced by the reaction $\mathrm{n}+\mathrm{p} \rightarrow \gamma+\mathrm{d}$ on the free protons of the liquid scintillator, were observed. The absolute detection efficiency for neutrons and for 4.44 and 2.2 MeV gamma's was measured and compared with Monte Carlo predictions. Fig. 12 shows the two gamma-lines and the comparison between experimental data and Monte Carlo.

\section{The streamer tube system}

Tracking in MACRO is performed with plastic streamer tubes. A preliminary description of the streamer tubes of MACRO has been given elsewherc [10]. They have been developed from the design used for the NUSEX underground experiment [11] and hadron calorimeters for LEP experiments [12]. The tubes operate in the limited streamer mode [13], and take advantage of the resistive cathode technique [14] to allow bi-dimensional readout on the same plane.

The tube system has a modular structure consisting of chambers, $3.2 \mathrm{~cm} \times 25 \mathrm{~cm} \times 12 \mathrm{~m}$ size, each containing eight individual cells. Each horizontal plane is made of 24 chambers, while the planes of the vertical and end walls are made of 14 chambers. The total number of wires for one supermodule is 5856. A picture showing some details of a streamer tube chamber is shown in fig. 13. The basic unit is an open cell profile extruded from $1.5 \mathrm{~mm}$ thick PVC. The single cell is $2.9 \times 2.7 \mathrm{~cm}^{2}$. The anode wire (silvered $\mathrm{Be}-\mathrm{Cu}$ ) has a diameter of $100 \mu \mathrm{m}$. Plastic inserts support the wire every $1 \mathrm{~m}$. The wires of each chamber are soldered to printed circuit boards at both ends. Three sides of the cell are coated with a low-resistivity graphite ( $\leq 1 \mathrm{k} \Omega$ /square) to perform the cathode function by the electrodeless shaping principle [15]. The field configuration is shown in fig. 14. This structure is inserted inside an uncoated PVC envelope (1.5 $\mathrm{mm}$ thick) and closed by two plastic end caps at the

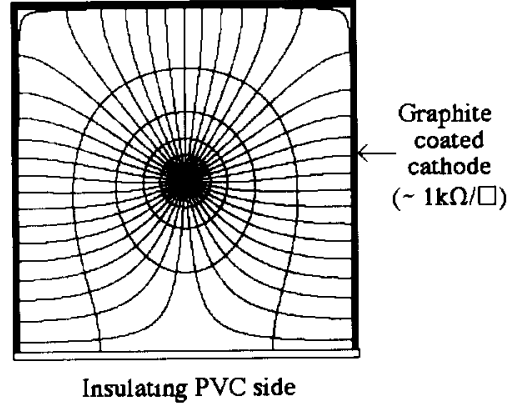

Fig. 14. Calculated electric field configuration in a streamer tube of MACRO.

ends. On one of the two end caps the high voltage connections to each wire and the ground connection to the graphite cathode are inserted. Both end caps have gas connections. Each wire behaves as a transmission line with a characteristic impedance of $330 \Omega$ and a propagation time of $\sim 3.3 \mathrm{~ns} / \mathrm{cm}$.

Particular care has been devoted to the gas seal: the end caps are both glued and heat sealed to the PVC envelope. The tightness of the chambers has been tested under low vacuum $\left(10^{-3} \mathrm{~atm}\right)$ just after construction, and again just before the installation in the detector. The thickness of the PVC envelope allows only a minimal diffusion of light gases: for $1 \mathrm{~atm}$ of helium the measured diffusion rate is $\sim 5 \times 10^{-6} \mathrm{~atm}$ $\mathrm{cm}^{-2} \mathrm{~s}^{-1}$.

For the horizontal planes, the two-coordinate readout is provided in one view by the wires and on the other by pick-up external strips placed at $26.5^{\circ}$ with respect to the wires. The streamer modules are placed upon the strip planes so that the insulating side of the active cell faces the strips directly. The pick-up strips are made of an aluminum ribbon ( $40 \mu \mathrm{m}$ thick) attached to a PVC foil ( $1 \mathrm{~mm}$ thick). On the other side of the plastic foil an aluminum sheet $(40 \mu \mathrm{m}$ thick) provides a ground reference electrode. A similar aluminum foil provides a ground on the opposite side of the streamer modules. The vertical streamer planes have only the wire readout. HV is distributed in parallel to the wires of each plane by distribution boxes. Each box contains a $1 \mathrm{M} \Omega$ limiting resistor/wire and a $2 \mathrm{nF}$ blocking capacitor to pick-up the current signal from the wire. The signals from the eight wires are sent to the front-end electronics through a connector inserted on each distribution box. A current limit of 10 $\mu \mathrm{A}$ is set for each plane.

Gas distribution is performed in parallel to all planes, where each plane is connected in series. INOX pipes are used to transfer gas from the mixing station to flow meters and then to the streamer modules. The gas volume of the active elements of the first supermodule is $\sim 59 \mathrm{~m}^{3}$. The first supermodule has been 


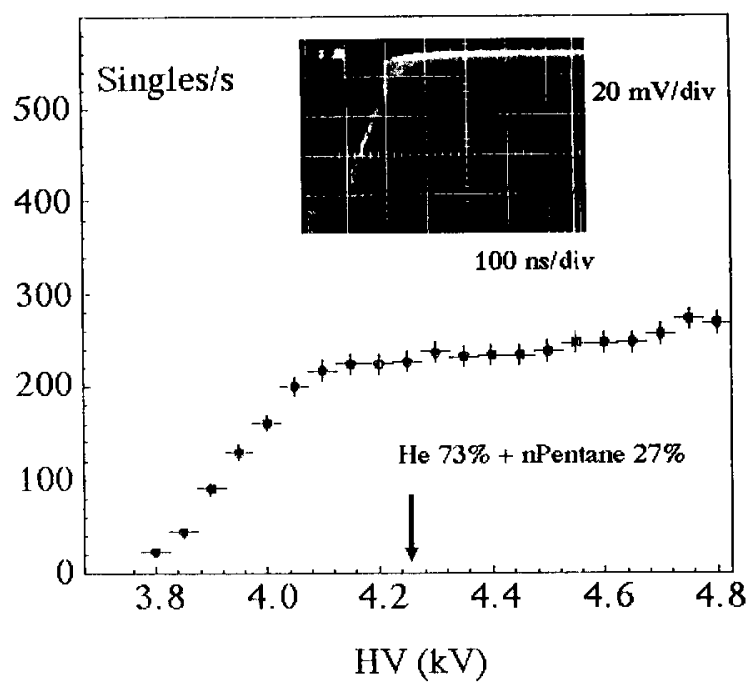

Fig. 15. Singles rate plateau as a function of high voltage and wire pulses ( $50 \Omega$ termination) for a streamer tube test chamber of MACRO (50 cm long).

operated with different gas mixtures. In a first run it was $\operatorname{Ar}(70 \%), \mathrm{CO}_{2}(5 \%)$ and n-pentane $(25 \%)$, obtained by bubbling a proper $\mathrm{Ar}-\mathrm{CO}_{2}$ mixture through n-pentane kept at $3^{\circ} \mathrm{C}$. In a second period, a mixture of Ar $(35 \%)$ and isobutane $(65 \%)$ was adopted. Eventually, He (73\%) and n-pentane (27\%) was chosen. This final mixture will allow the exploitation of the Drell effect [16] for slow monopole detection. Gas flow is kept at a rate of one change of the whole volume every five days, and the pressure inside the chambers is maintained at a few mbar above atmospheric pressure.

Fig. 15 shows the measured singles rate versus high voltage for a test chamber exposed to beta rays using the $\mathrm{He}+\mathrm{n}$-pentane gas mixture. The plateau corresponding to full efficiency noiseless operation is at least $700 \mathrm{~V}$ wide. The wire pulses at $250 \mathrm{~V}$ above the knee of the single's rate plateau are shown. At this operation point, very weakly ionizing particles can trigger a streamer with reasonable efficiency: $\sim 20 \%$ for single ionization electrons has been measured [17]. The maximum drift time in a tube is $\sim 600 \mathrm{~ns}$. The dead region along the wire induced by the streamer discharge is a few $\mathrm{mm}$, for a time interval of $\sim 300 \mu \mathrm{s}$. Tests performed with relativistic ion beams [18] have shown that large ionization induces an increase of total streamer charge which is logarithmically dependent on $\mathrm{d} E / \mathrm{d} x$ for $\mathrm{d} E / \mathrm{d} x>\mathrm{d} E / \mathrm{d} x_{(\mathrm{m} \text { 1.p.) }}$.

\subsection{The wire readout electronics}

The readout card consists of eight channels for processing the signals from the wires of a MACRO eight-fold streamer tube module. The card is directly connected to the HV enclosure of the tube with a 16 pin connector. In addition, to keep it firmly connected to the tube, its width is only $140 \mathrm{~mm}$ and it is inserted between two plastic guides fixed to the enclosure. With

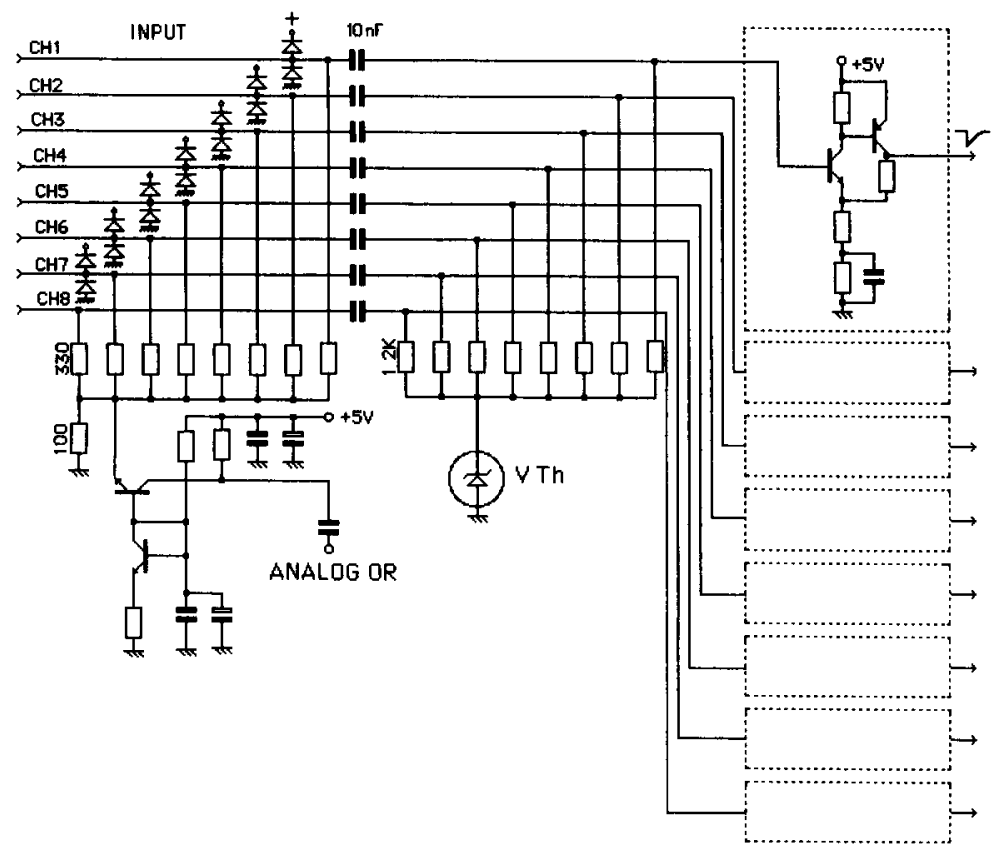

F1g. 16. Eight amplification input stages and the "analog OR" for the streamer tube wire readout card. 
this mechanical solution it is quite easy to interconnect the cards with a short flat cable.

In fig. 16, the eight amplification input stages (one for each wire) and the "analog OR" are shown. The input signals $\left(V_{\mathrm{n}}>50 \mathrm{mV}\right.$ on $330 \Omega$ ) are connected to two amplifiers. The first amplifier is fed by a signal derived from the resistive chain formed by a $330 \Omega$ resistor in series with the input impedance $(15 \Omega)$ of the first stage. The analog signal from this amplifier is sent via a twisted pair cable to ADCs and TDCs (QTP modules) and to the trigger logic. The individual preamplifier for each wire has an input threshold of 40 $\mathrm{mV}$. Its output is TTL converted and feeds the digital part of the card.

In fig. 17 , the card digital section is shown. The TTL pulse widths are $10 \mu \mathrm{s}$ for fast particle tracking (fast chain) and $550 \mu$ s for slow monopoles (slow chain). The eight signals coming from the $10 \mu \mathrm{s}$ one shot are ORed: the resulting signal is available (DIGOR BOARD FAST OUT) for further triggers. An additional OR signal is made with the previous card OR (IN/OR): it is available on the card connector and is sent via the interconnection flat cable to the next card (DIGOR/BUS). The one shot outputs of each chain are connected to two parallel-in serial-out shift registers. The serial input of any shift register comes from the previous card while the serial output feeds the following card. In the horizontal planes, 24 cards are connected in series and there are 14 in the vertical planes. The load, clock, shift-in, shift-out sig-

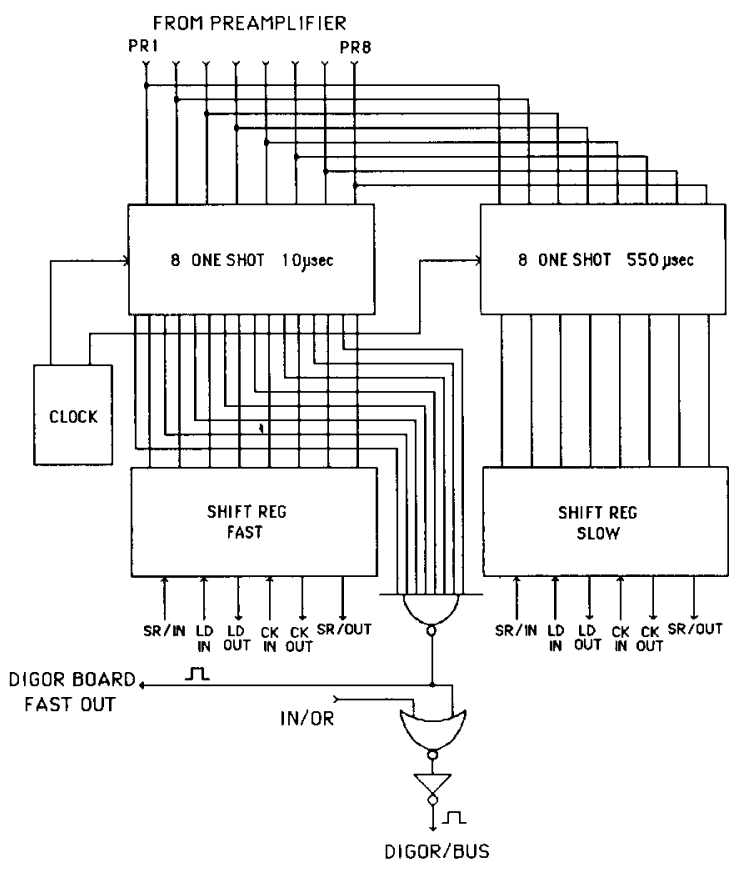

Fig. 17. Digital layout of the streamer tube wire readout card.

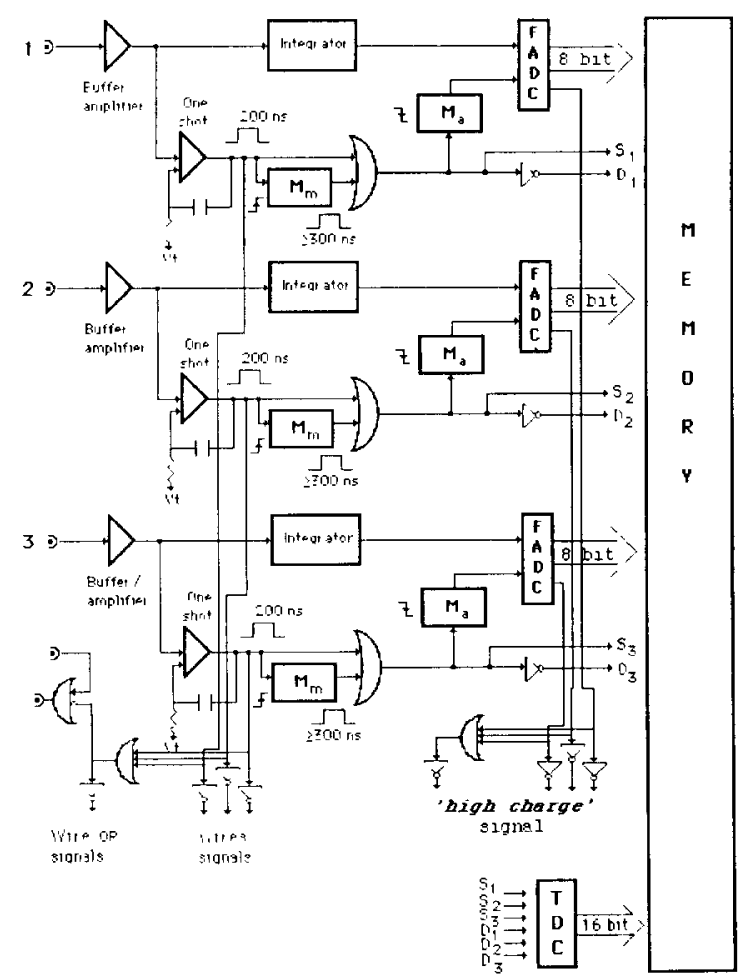

Fig. 18 Electronıc scheme of the QTP board.

nals are fed to each card by drivers. The supply current for each card is $15 \mathrm{~mA}$. The hybrid card has dimensions of $140 \times 80 \mathrm{~mm}^{2}$.

\subsection{The charge and time processor for streamer tubes (QTP)}

The sensitivity of streamer tubes to single ionization electrons ensures a good response down to $\beta \sim 10^{-4}$. A vertically moving monopole with $\beta \sim 10^{-4}$ crosses a single streamer tube layer in about $1 \mu \mathrm{s}$, and the whole apparatus in $150 \mu \mathrm{s}$. The crossing time increases with the track angle. A maximum time acceptance is set at $600 \mu \mathrm{s}$, which gives a complete angular acceptance.

A QTP system (charge and time processor) has been designed to provide signals for triggering and to measure arrival time, duration, and charge of streamer tube signals. The system consists of three electronic circuits:

1) QTP cards to process signals from the streamer tubes and to store charge and time in a cyclic memory (see fig. 18).

2) A transceiver card to manage the QTP's Eurocard crates.

3) A CAMAC processor to read stored information, select data in the $600 \mu \mathrm{s}$ time window and transmit them to the MicroVAX. 
Each QTP channel serves 32 wires of a horizontal plane, so a QTP card having three channels serves a half plane. A central module needs 20 cards in an Eurocard crate. Furthermore, each QTP channel serves 16 wires in a vertical plane, and 14 QTP cards are needed to read all the vertical planes of a supermodule. For trigger purposes, each board presents three outputs and their OR. Also, three "high charge" outputs and their OR are available to trigger high charge events. A 16 bit free counting 6.6 MHz TDC gives the time of the rising edge and falling edge of the shaped signal with a precision of $150 \mathrm{~ns}$, which is about the time resolution of the streamer tube system. For each channel, a linear integrator and 8-bit $20 \mathrm{MHz}$ FADC provides the charge measurements in a range from 2 to $3000 \mathrm{pC}$ (fig. 18). Four regions with different conversion slopes provide a large dynamic range without losing sensitivity for small signals. Hence, the streamer signal arrival time, its duration and its charge are measured and stored in a cyclic memory, 63 bytes long. This information permits discrimination between relativistic muons and slow crossing particles as well as muon initiated showers and high activity events inside MACRO.

An Eurocard crate can contain up to 20 QTP boards managed by a control board called a transceiver. This card generates the $150 \mathrm{~ns}$ common clock to all QTPs and to the CAMAC processor reading the crate, which synchronizes the whole system. In addition, the transceiver permits the calibration of the FADCs, generating a sample of signals and sending them to the QTP input. Finally, the transceiver controls the supply voltage and the flow of data between QTP and CAMAC readout card. The system was successfully tested on the first MACRO module and has been operating since October 1989.

\subsection{The strip readout electronics}

The strip readout cards [19] are connected to the strips via twisted pairs soldered directly on the strips (signal and ground). Every card reads 32 strips. Induced input signals are fed to a hybrid chip containing two D779 CMOS integrated circuits. Each D779 serves four inputs and consists of an amplifier, a discriminator, a digital one shot, a shift register plus a digital $O R$ (DigOR), which groups four channels as shown in fig. 19. The width of output signals is determined by a 4.43 $\mathrm{MHz}$ clock. Five clock cycles fix the shaping time to be about $580 \mu \mathrm{s}$ to allow detection of slow monopoles (down to $\beta \sim 10^{-4}$ ). The serial output of each register is connected to the serial input of the next, achieving a 32-bit shift register per card (slow chain) having a memory of about $580 \mu \mathrm{s}$. To reduce background contamination on relativistic events $(\beta \sim 1)$, another shift register chain (fast chain) is added by reshaping the

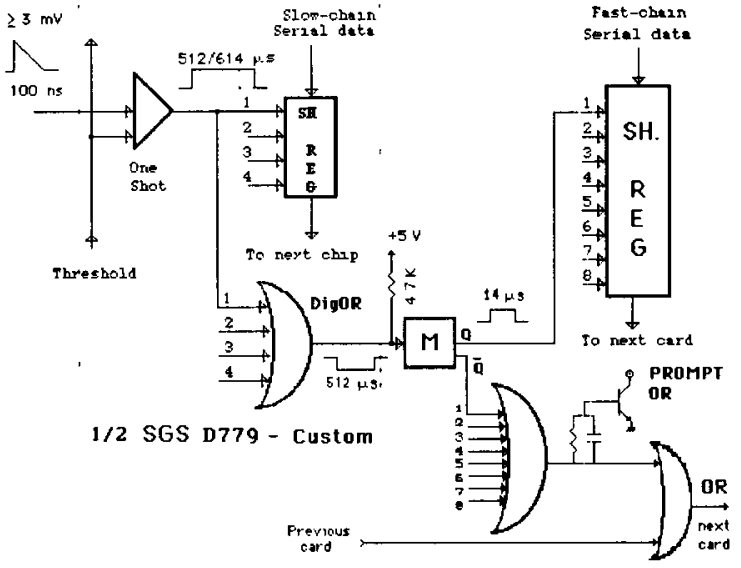

Fig. 19. Schematic of a single readout channel of the streamer tube strip electronic card.

DigOR pulses to $14 \mu \mathrm{s}$. A board OR is also available for general purposes. A $30 \mathrm{~Hz}$ reset signal is fed to all the D779 to perform the offset null of the sense amplifiers. This reset produces a dead time in the D779, which is less than $1 \%$. The recovery time of the threshold setting after a reset pulse is negligible. The probability to have electronic cross-talk when a pulse is fed into the hybrid input stage is also negligible. The hybrid chip input impedance is $\sim 25 \Omega$ which can be matched to the characteristic impedance of the strip line by adjusting by an external resistor.

The strip readout efficiency has been studied by analyzing two sets of data, corresponding to different values of the threshold settings: 1.8 and $2.4 \mathrm{mV}$. For the two samples, the readout efficiencies have been found to be $98 \%$ and $96 \%$, respectively. In MACRO, the strips have different lengths but even if there is no sharp separation between short and long strips, two different regions can be singled out: a region of shorter strips (about 400 strips per supermodule whose length is less than $7.5 \mathrm{~m}$ covering about $35 \%$ of the area), and one of longer strips (240 strips whose length is greater than $7.5 \mathrm{~m}$ ). The analysis shows full readout efficiency on short strips for both values of the threshold setting and a very high long strip efficiency: $97 \%$ at $1.8 \mathrm{mV}$.

\subsection{Streamer tube triggers}

The streamer tube is a low noise device. Its signal rate is dominated by ionizing particles due to radioactive decays. In the MACRO building phase, special care was devoted to choosing materials that reduced this source of noise to a very low level: $40 \mathrm{~Hz} / \mathrm{m}^{2}$. This low rate permits the use of trigger algorithms without tracking requirements. The inputs to the trigger circuits are the $\mathrm{OR}$ of the discriminated signals coming from a $12 \times 12 \mathrm{~m}^{2}$ plane of horizontal streamer tubes 
or $3.5 \times 12 \mathrm{~m}^{2}$ vertical streamer tubes. With the gas mixture used, the signals have a time jitter smaller than $600 \mathrm{~ns}$ and the resulting tracking system has a time resolution of $150 \mathrm{~ns}$.

The circuit used in the MACRO apparatus for triggering on fast particles is based on EPROM components and works with standard TTL positive logic [20]. This circuit manages up to 26 lines from independent counters and has high triggering capacity and flexibility. For each module, a counter is made by ORing the signals coming from the streamer tubes of the same plane. To reduce the loss in acceptance, the resulting signals are individually split and overlapped with those coming from the corresponding plane of the adjacent modules, respectively. Each logical unit has 26 counter lines, whose signals are TTL formatted with a time length adjustable between 900 ns and 2500 ns. These signals are handled as binary words, each of them representing the address of a memory location inside the EPROM. Each EPROM memory location holds an 8-bit word coded according to whether the address corresponds to a good trigger configuration. Since the commercially available EPROMs have a maximum of 17 input lines, three EPROMs have been used with the 26 counter lines partitioned between them. The logical functions are divided into an horizontal group (12 lines) and a vertical one (14 lines). The horizontal group is fed to one EPROM and the vertical group to another, taking care respectively of particles able to trigger the apparatus with horizontal or vertical counters only. A third $32 \mathrm{k} \times 8$-bit EPROM is used to take care of those inclined tracks for which the triggering condition is achieved by a combination of horizontal and vertical signals, even though neither horizontal nor vertical signal combinations produce a trigger alone.

All the input signals are sampled at a $3.3 \mathrm{MHz}$ frequency in order to check for valid coincidences within each sample. This is achieved by a free running clock, c (150 ns pulses, 300 ns period), strobing four 8-bit latches as shown schematically in fig. 20 . In addition to clock $\mathrm{c}$, a clock $\mathrm{d}$, (equivalent to $\mathrm{c}$ ) and a clock e, (70 ns pulses, $300 \mathrm{~ns}$ period), both driven by c, are used. The clock $d$ is AND-gated with latch outputs. The EPROMs are enabled to READ continuously. The final outputs of the third EPROM are ANDed with clock e that is inverted and delayed to cope with the signal transit time across the three EPROM system. To avoid any possible trigger loss, the pulses of the free running clock $c$ have to agree with the access time of the EPROMs and with the signal overlapping. The signal formation length depends on the length of the jitter. For the same event, it is possible to have one or more trigger signals, depending on the phase between the clock $c$ and the input signals. A retriggerable one-shot at the end of the line sums these multiple triggers into a single longer pulse. To continuously check the apparatus performance and provide a diagnostic, the latch outputs are sent to a $2 \times 16$ bit CAMAC register, strobed by the EPROMs trigger output. EPROM coding depends on the triggering criteria for which the circuit is used.

The triggering conditions chosen for the first supermodule of the MACRO apparatus are: (Majority coincidence of six out of ten horizontal streamer planes) OR (Majority of five out of eight contiguous horizontal planes, first and the tenth planes excluded) OR $(3 / 10$ horizontal AND 3/6 vertical west planes) OR (3/10

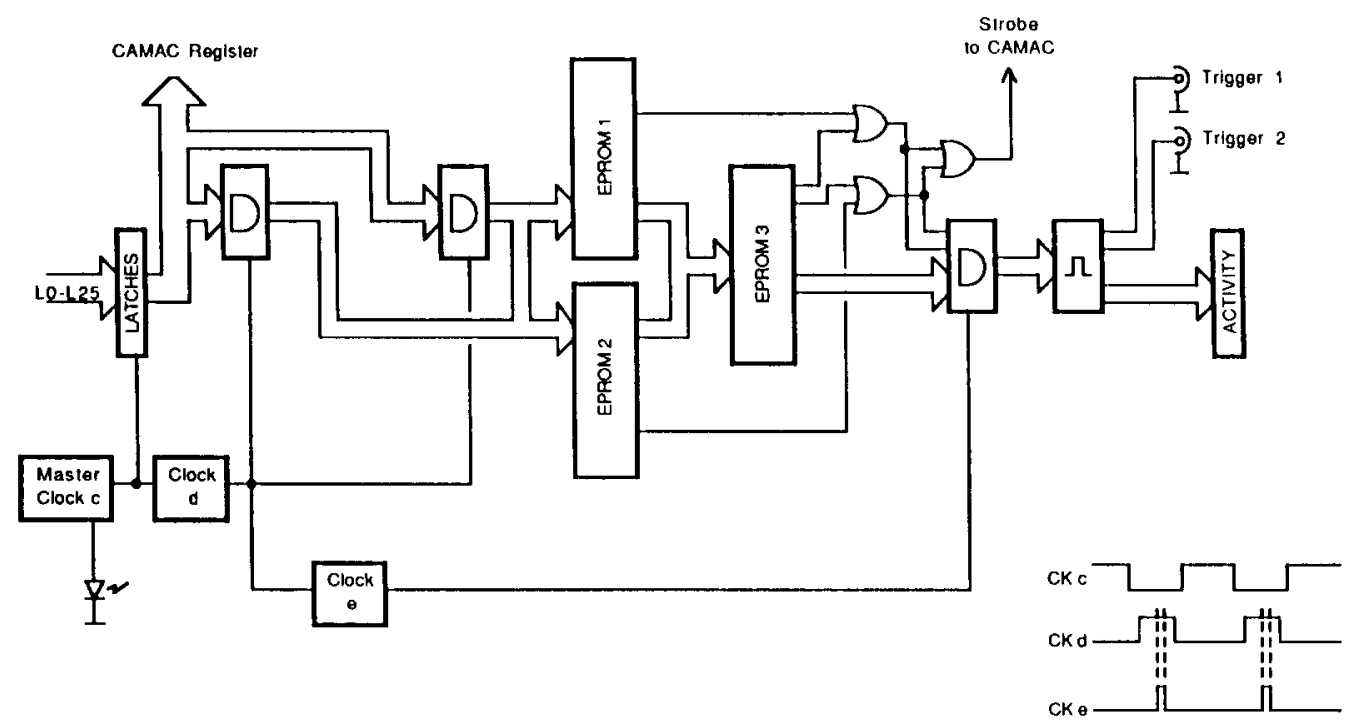

F1g. 20. Schematic overview of the streamer tube trigger circuit 
horizontal planes AND 3/6 vertical east planes) OR (3/6 vertical east planes AND 3/6 vertical west planes) OR (5/6 vertical east planes) OR (5/6 vertical west planes). This is the triggering function using streamer tubes only. The total number of logical combinations is about 5500 and is well matched by the trigger circuit capability. All of these conditions can be changed by recoding the EPROMs.

In addition to the fast particle streamer tube trigger in MACRO, there are two independent slow particle trigger systems for horizontal and vertical streamer tube planes. A complete description of the trigger from horizontal tubes is given in ref. [21]. The purpose of the streamer tube slow particle trigger is to distinguish signals which may be produced by a monopole from random background signals occurring during the long gate time $(\sim 0.5 \mathrm{~ms})$. Since limited streamer tubes are essentially noiseless, the only background is natural radioactivity events which occur at a rate of $\sim 40$ $\mathrm{Hz} / \mathrm{m}^{2}$ in the MACRO detector. In contrast, a massive slow particle would traverse the detector in a straight line and at constant speed. Hence, it is possible to trigger on events in which the vertical coordinates of the hit planes are temporally aligned. The trigger for the horizontal planes acts as the OR of 320 delayed coincidences with a resolving time of $2 \mu \mathrm{s}$. Each coincidence requires the time alignment of at least seven hit planes corresponding to a particular time-of-flight window. The triggering circuit is based on 20 custom gate arrays, each with the equivalent of 6000 gates. For the vertical streamer planes, timing requirements alone are not sufficient to form a trigger since the time-of-flight between the inner and outer vertical planes is short except for very low beta particles. Hence, in addition to a loose time alignment, the vertical plane trigger requires spatially aligned hits. Both types of trigger units (horizontal and vertical) produce a trigger rate of $10^{-2} \mathrm{~Hz}$. The product of the electronics efficiency times the acceptance is $70 \%$ of a full $4.8 \times 12 \times 12 \mathrm{~m}^{3}$ sensitive box for $10^{-4}<\beta<1$. Lower thresholds (and higher acceptance) will be used in the final MACRO configuration.

\subsection{Space and angular accuracy of the tracking system}

Tracking precision is important in determining the true directions of penetrating particles to study muon astronomy and angular distributions of muons. In addition, it is of primary importance for selecting candidate slow particles (e.g., monopoles, nuclearites, etc.). In this latter case, since a relatively long time window is used $(500 \mu \mathrm{s})$, it is mandatory to recognize a candidate track among the natural radioactivity noise. Spatial resolution depends on the granularity and performance of both wire and pick-up strip views. More precisely, the localization of the track is defined within a cluster, which is the group of tracking elements fired when a particle crosses a plane. The size of the induced signal on adjacent strips depends on the detector design and gas mixture, and its importance depends on the electronic threshold of the readout elements. The cluster width distribution is closely related to the spatial accuracy obtained in the stereo view. Moreover, track reconstruction is affected by particle interactions just outside and within the apparatus. In fact, electromagnetic processes leaving energy deposits in the streamer

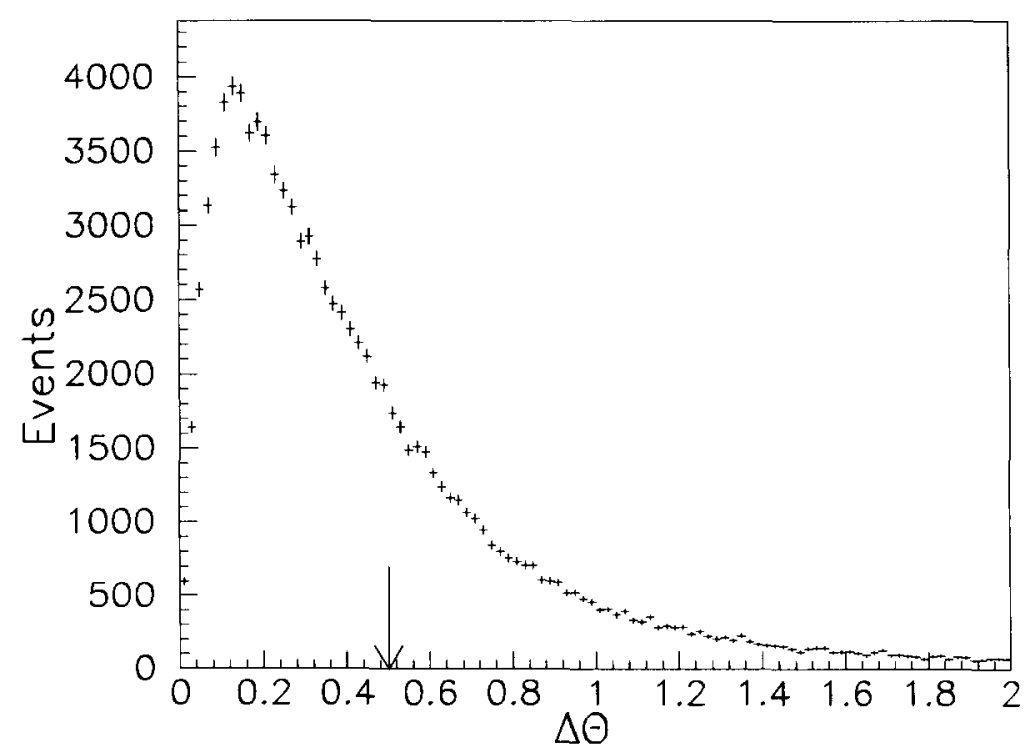

Fig. 21. Angular resolution of the muon tracking system as derived from the study of track slopes for the wire and strip views. 
tubes near the cell that the particle crosses can enlarge the cluster width. The average cluster widths have been measured to be $4.5 \mathrm{~cm}$ in the wire and $8.96 \mathrm{~cm}$ in the strip view, corresponding to a mean occupancy of 1.45 and 2.75 elements, respectively. Space resolution achieved without any selection on the tracks is $\sigma(\mathrm{w})=$ $1.1 \mathrm{~cm}$, and $\sigma(\mathrm{s})=1.6 \mathrm{~cm}$, where $\sigma(\mathrm{w})$ refers to the wires and $\sigma(\mathrm{s})$ to the strips. These numbers refer to the residuals obtained by subtracting the cluster centers from the position of the straight line passing through the other hits belonging to the track. This result confirms that the tracking system is operating correctly.

The muon tracking procedure consists of a pattern recognition section, based on the search for a set of aligned points. Starting from these selected hits we perform a linear fit and calculate track parameters and their errors. For each hit element, the actual coordinate is assumed to be uniformly distributed within the entire cluster width. The angular resolution depends on the cluster widths and on the track length. The average errors on the slopes are $\sigma(\mathrm{w})=0.14^{\circ}, \sigma(\mathrm{s})=$ $0.29^{\circ}$ (only tracks with 4 or more hits are kept). The angular resolution related to these values is reported in fig. 21 where it is shown that $68 \%$ of the tracks fall within $0.48^{\circ}$ of the original direction. It should be noted that for muon physics, the instrumental angular resolution is only one component of the uncertainty on the original direction of the muons. The main contribution comes from the multiple coulomb scattering due to the passage of the muons in the rock overburden. The Earth's magnetic field and the incident particle's transverse momentum in the normal production of the muons from pion and kaon decay are completely negligible.

The measurement of the overall resolution has been performed using the relative angle of muons in double muon events and is shown in fig. 22 . The $68 \%$ width of the distribution is $1.35^{\circ}$, hence, the resolution on single tracks, $(\sqrt{2}$ better $)$, turns out to be $0.95^{\circ}$.

\section{The track-etch detector}

The main purpose of the MACRO track-etch detector is the search for magnetic monopoles [22]. The detector is placed horizontally in the middle of the MACRO structure. Vertical walls on the east and north sides of MACRO are being added for the final configuration. The horizontal track-etch detector is organized in 48 "trains" per supermodule; each train has 47 "wagons" of $25 \times 25 \mathrm{~cm}^{2}$ in size. A "wagon" contains three layers of CR39, $1.4 \mathrm{~mm}$ thick, $1 \mathrm{~mm}$ thick aluminum absorber, and three layers of LEXAN, $0.2 \mathrm{~mm}$ thick. These layers are placed in an aluminum bag filled with dry air. Each "wagon" can be inserted or removed by sliding it on rails. The passage of magnetic monopoles is expected to cause structural damage in the CR39. Chemical etching should result in collinear etch pits of equal size on both faces of the sheet. The track-etch detector will be used mainly for confirmation, when "triggered" by a signal in the scintillation counters and/or the limited streamer tubes. For these candidate events, a search is performed for a monopole track in the CR39 around the expected position, defined to within a few square centimeters.

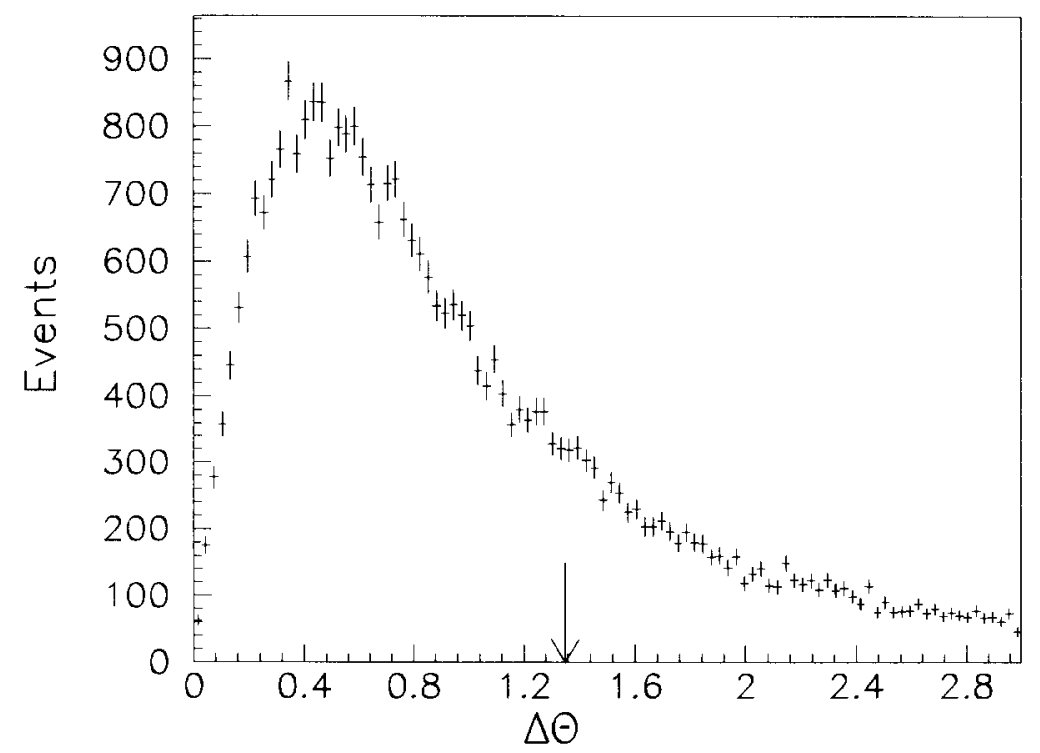

Fig. 22. Overall angular resolution of the muon tracking system as derived from the study of di-muon events. 
The interesting "wagon" is extracted and one layer of CR39 is heavily etched. A scan for holes is performed with low magnification optical methods. The other two sheets of CR39 are used for confirmation of any candidate monopole track found in the heavily etched layer. The relative position of the monopole tracks in the different layers of CR39 can be determined with a precision of better than $100 \mu \mathrm{m}$. The technique has been tested and shown to be viable.

In collaboration with the INTERCAST Company of Parma, Italy, manufacturer of CR39 mainly for sun glasses, the quality of the polymer was improved in order to achieve lower detection threshold, higher sensitivity in a larger range of energy losses, higher quality of the post-etched surface after prolonged etching, stability of the sensitivity of the polymer over longer periods of time (several years), and uniformity of sensitivity for mass-produced sheets. After a number of tests, a first batch of 1000 CR39 sheets, each $98 \times 98$ $\mathrm{cm}^{2}$ in size, was made by INTERCAST. These were used for the first supermodule. Further improvements came from the use of a different catalyzer (IPP instead of CHPC), a different curing cycle, and a number of extra precautions. The present discussion will be restricted mainly to two types of CR39: EN3 (first batch) and L6 (later batches) that are already installed in MACRO.

The bulk etching rates of several samples of CR39 of different chemical composition and made with different curing cycles were measured by etching in a solution $6 \mathrm{~N}$ of $\mathrm{NaOH}$ at $70^{\circ} \mathrm{C}$ for successive etching times of $5,10,15,20$, and $40 \mathrm{~h}$. The thicknesses of the sheets were measured with a precision comparator after each etching period. Generally, the thicknesses increase in the first five hours of etching; then they decrease regularly. The slope of the decrease is the bulk etching rate. The increase in thickness at small etching times is connected with a swelling of the plastic, due to the combined effect of water and temperature. The bulk etching rates were determined by fitting the measured data after the first $10 \mathrm{~h}$ of etching. The results are given in table 1; errors include statistical and systematic uncertainties. The EN3 type has the larger etch rate $\left(v_{\mathrm{B}}\right)$. The post-etching surface quality

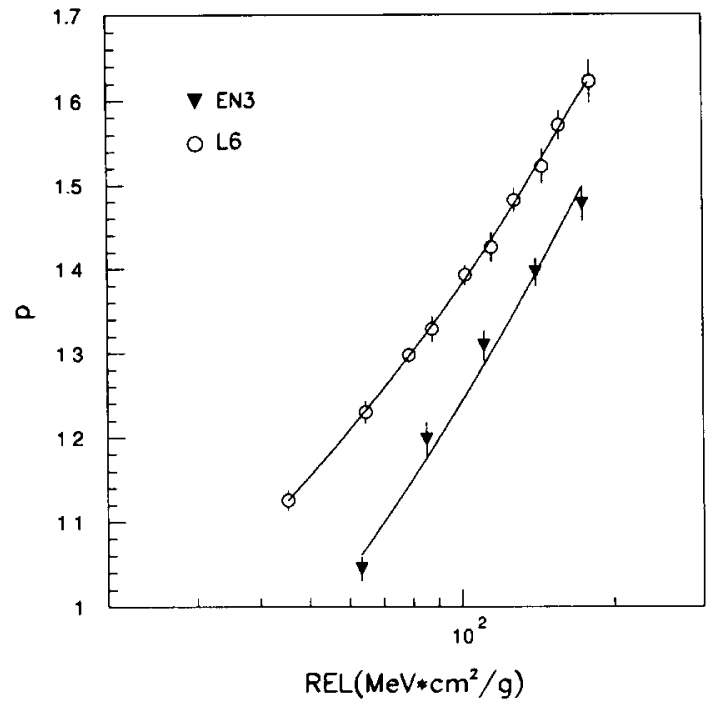

Fig. 23. Measured values of the reduced etch-ratio $p=v_{\mathrm{T}} / v_{\mathrm{B}}$ vs REL for EN3 and L6 types.

and transparency decrease from L6 to EN3. This suggested the convenience of the use of the IPP catalyzer instead of the CHPC catalyzer. After a number of tests, we limited the mass production to the L6 type.

In order to calibrate the CR39, stacks were exposed to ions of $585 \mathrm{MeV} /$ nucleon at the Berkeley Bevalac. The EN3 and L6 samples were 1.5 and $0.5 \mathrm{yr}$ old, respectively. After production, they were stored in aluminum bags filled with dry air in the Gran Sasso underground laboratory. A stack was typically made of 6 CR39 sheets, a copper absorber $1.4 \mathrm{~cm}$ thick, followed by ten sheets. All the CR39 sheets were $13 \times 7$ $\mathrm{cm}^{2}$ in size, and $1.4 \mathrm{~mm}$ thick. The sheets located after the absorber also detected lower $Z$ fragments from the original ions. After $45 \mathrm{~h}$ of etching in $\mathrm{NaOH}, 6 \mathrm{~N}$ at $70^{\circ} \mathrm{C}$, the surface areas of the etch-pits were measured with an Elbek image analyzer. From these measurements and the measurement of $v_{\mathrm{B}}$, the reduced etch ratio, $p=v_{\mathrm{T}} / v_{\mathrm{B}}$, was calculated for each detected charged fragment [23]. The values of $p$ obtained are plotted versus restricted energy loss (REL) in fig. 23 for both EN3 and L6 types. The continuous lines are

Table 1

Chemical composition of two types of CR39, the maximum temperatures reached in the curing cycle and the measured bulk etching rates

\begin{tabular}{llllll}
\hline Type & Date & Catalyzer & Additives & $\begin{array}{l}\text { Max. temp. in } \\
\text { the curing cycle } \\
{\left[{ }^{\circ} \mathrm{C}\right]}\end{array}$ & $v_{\mathrm{B}}[\mathrm{mm} / \mathrm{h}]$ \\
\hline EN3 & Jan. 88 & CHPC & DOP & 80 & $1.21+0.02$ \\
L6 & Jul. 88, & IPP & Naugard & 95 & $1.16+0.02$ \\
& Mar. 89, Jan. 90 & & Antioxidant & \\
\hline
\end{tabular}


the result of a best fit to the experimental data. The extrapolation to $p=1$ of each curve yields the threshold values, REL $=27$ and $53 \mathrm{MeV} \mathrm{cm} / \mathrm{g}$ for $\mathrm{L} 6$ and EN3 types, respectively. These values correspond to $Z_{\text {eff }} / \beta=5.1$ and 7.3 , respectively. The EN3 type should be sensitive to magnetic monopoles with unit Dirac charge having $\beta>1.8 \times 10^{-3}$ and also for $2.6 \times 10^{-5}$ $<\beta<1.7 \times 10^{-4}$ if nuclear energy losses can be detected in CR39. We are studying experimentally the response of CR39 in the very low $\beta$-region.

Samples from different sheets of the same batch were exposed to $\mathrm{Ne}$ ions of $585 \mathrm{MeV} /$ nucleon and etched together. The $p$-values obtained for each sheet indicate a good uniformity of sensitivity for sheets of the same batch. We checked that the CR39 of the EN3 type shows no significant loss of sensitivity near threshold after $1.5 \mathrm{yr}$ of installation in the MACRO detector. The result suggests that the sealing of the CR39 in dry aluminum bags filled with dry air at $15^{\circ} \mathrm{C}$ (MACRO environment) are good storage conditions.

Preliminary results indicate that both EN3 and L6 types of CR39 are adequate for the search for magnetic monopoles in MACRO $[24,25]$. The relatively large difference in threshold between samples of EN3 and L6 makes only a relatively minor effect on the $\beta$-threshold of monopoles.

\section{On-line data-acquisition system}

The MACRO data-acquisition system [26] has been designed taking into account the following requirements:

1) The system must be modular and must consist of a network of microcomputers to match the apparatus modularity. In this way the system size can be tailored to the real necessities.

2) The system must allow easy access from remote locations to each computer or microcomputer in order to control the apparatus.

3) The system must be based on commercial products, both for software and for hardware, in order to be easily maintained.

Taking into account these requirements, we have chosen a system based on a MicroVAXII network (Ethernet/DECNET). The MicroVAXes are running a VAXELN system. VAXELN is a Digital Equipment software product for the development of dedicated, real time systems for VAX processors. A central VAX, running under the VAX/VMS operating system, is used as file server and interface with respect to the user's world. The "CERN-Fisher CAMAC system crate", that uses the CES 2280 Qbus-CAMAC interfaces with the DMA controller 2281, has been chosen as the main hardware standard. The general layout of the acquisition system is shown in fig. 24. For the full

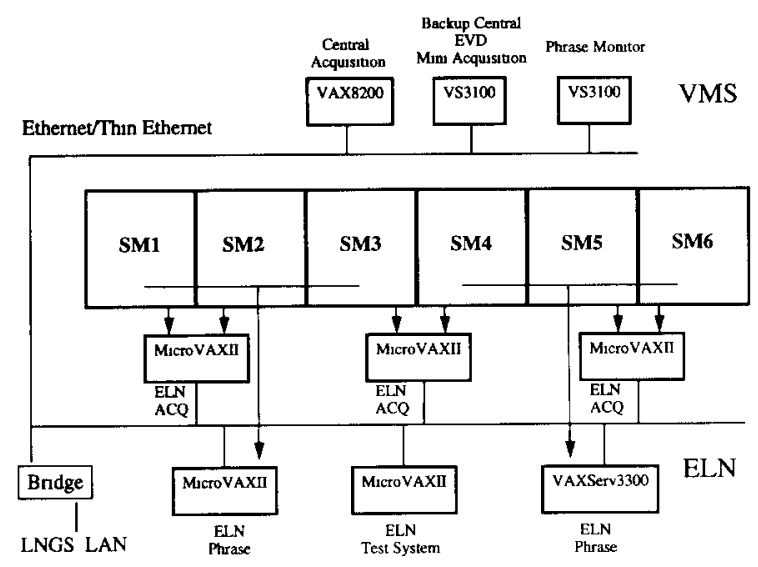

Fig. 24. General layout of the global data acquisition system in MACRO. SM1 to SM6 represent the six supermodules of the final MACRO detector.

MACRO detector, three MicroVAXes will control three groups of two supermodules each. Each MicroVAX is connected to two CAMAC parallel branches. Two additional MicroVAXes will act as the supervisors for the neutrino events from stellar collapse. The raw data logging is performed on the VAX8200 disks and the amount of mass storage (3 Gbytes) allows the data to be available for a few weeks to allow for a fast analysis and copying to tape.

All the computers are connected via Ethernet under DECNET protocol. This choice does not produce any limitation on the data throughput. We have obtained, in absence of high software overload, a data throughput of more than $300 \mathrm{kbyte} / \mathrm{s}$ between two $\mathrm{Mi}$ croVAXes in VAXELN, and more than $150 \mathrm{kbyte} / \mathrm{s}$ between a MicroVAX in VAXELN and the VAX8200. If the data are logged on a VAX8200 disk, the data throughput is of the order of $40 \mathrm{kbyte} / \mathrm{s}$. An advantage of this network solution is the reduction of cables, since it is not necessary to have all the crates in the same location. In the future some VME electronics will be included in the acquisition. In this case, the readout will be done using a VME board with a MicroVAX CPU and ethernet interface.

The MACRO local area network (LAN) is connected to the general LNGS LAN by a DEC Bridge. The LNGS LAN is composed of segments linked via optical fibers running for about $6 \mathrm{~km}$ between the underground laboratory and the external laboratory, where the general computing facilities are located. From the external laboratory, it is possible to have access to the DECNET wide area network (WAN). Utilities have been implemented to help control and debug the apparatus. In fact, during the acquisition, a user from any DECNET node can request a copy of the raw data buffer from a given MicroVAX or from 
the central VAX as well as require the execution of CAMAC operations by the use of remote procedure calls (RPC).

\subsection{The VAXELN system for the MicroVAXes}

VAXELN has the following advantage with respect to the VMS operating system:

1) optimized performance for real time operations due to the small overhead of the operating system;

2) powerful and efficient message exchange facilities that are useful for building multi-job applications, even if the jobs are running on different machines;

3) ease of writing device drivers and working with peripherals. Device drivers can be written using high level languages (EPASCAL or C).

The VAXELN system for MACRO consists of seven user jobs running with different priorities. The system includes four system jobs such as the Ethernet driver, the console driver, etc. In each job many concurrent subprocesses can run with different priorities; in our system more than seventeen subprocesses run concurrently. The number of subprocesses depends on the number of activated DECNET connections. The software has been written in the VAXELN PASCAL version of EPASCAL.

The CAMAC input/output can be done using a list of CAMAC operations contained in a file on the VAX8200. This is the normal way to handle CAMAC during the data taking. Moreover, the access can be done by using a standard ESONE CAMAC library, either directly inside VAXELN, or from a computer in the DECNET network via remote access routines.

The data flow after the occurrence of a trigger is shown in fig. 25. The exchange of data between jobs is done using the VAXELN message facilities. After a trigger interrupt the data are read inside the Event_In job using a memory region mapped as a VAXELN message. A concurrent subprocess, running at lower priority, provides the message mapping and the message routing. The time needed for these operations is 4 ms for a buffer of 100 kbyte. Two memory regions are allocated as data buffers to minimize the dead time. The overhead, due to this architecture, sets an absolute limit on the maximum peak acquisition rate of $\sim 90 \mathrm{~Hz}$.

In the MicroVAX used to monitor low energy neutrino events, the data are buffered (16 events) to improve the performance. In the same job, a periodic readout of equipment is performed (e.g. voltages, currents, scalers). These data are inserted in the same stream as the normal events but with a different identifier. The data can be sent either to a job, dedicated to the data reduction and data forwarding to the VAX8200, or to the Event filter and pre-analysis job. In the Data_ Reduction job, data are zero suppressed,

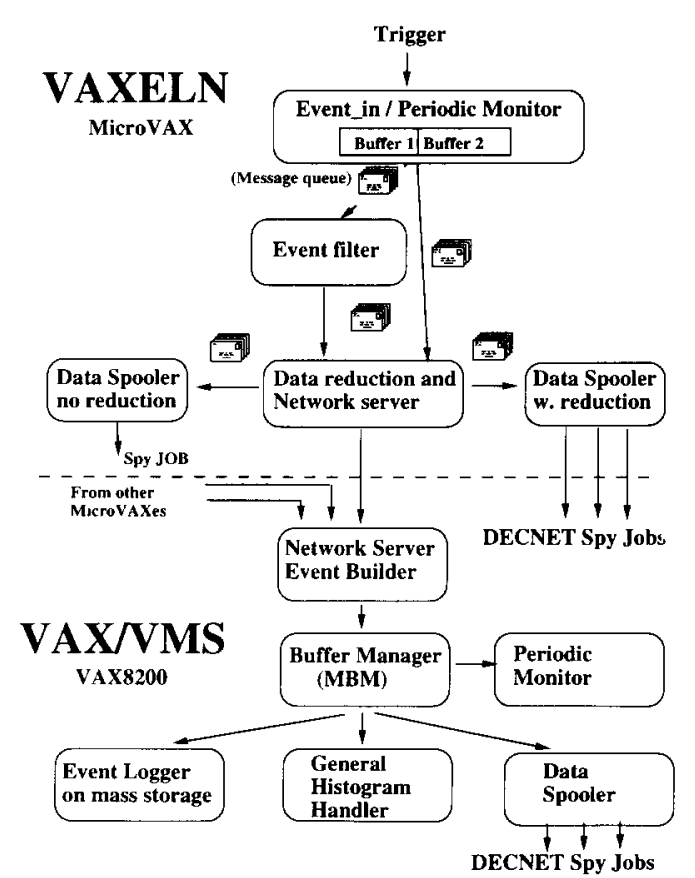

Fig. 25. Data flow from a MicroVAX to the VAX8200 following a trigger.

if necessary, and the waveform data are compressed according the Huffman compression algorithm [27]. The event filter job decides whether the event should be discarded after a pre-analysis based mainly on the tracking. In the acquisition with the first supermodule, however, all events are accepted. Also, the data are sent to two data spooler jobs, which run at low priority. The first copies the data before reduction, the second copies the data after reduction. Users from remote DECNET nodes can ask for a copy of the data buffer by requesting a DECNET connection to a spooler job. A centralized job handles the commands coming from the central VAX and routes such commands to the appropriate VAXELN job (fig. 26). This job also supports the ESONE remote CAMAC procedures calls coming from the main acquisition program.

\subsection{The VAX/VMS acquisition system for the VAX 8200}

The VMS part of the acquisition system performs the following functions:

1) Network server and I/O server for the ELN systems running on the MicroVAX network;

2) router of the directives to the MicroVAX network;

3) router of the user directives to the subprocess components of the VMS system;

4) handler of the general histogramming; 

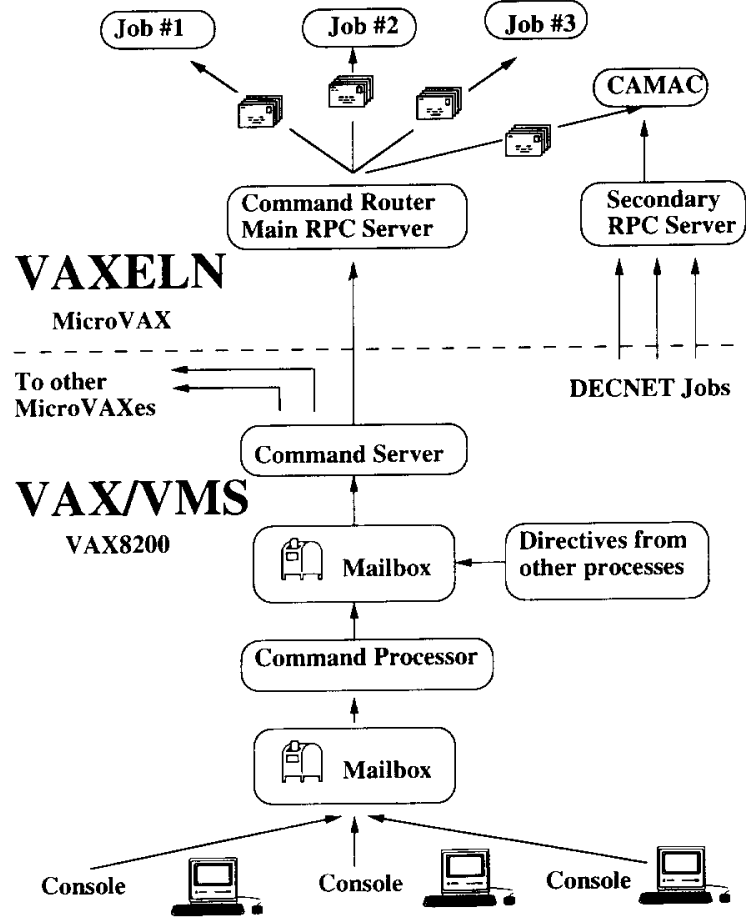

Fig. 26. Command flow from the VAX8200 to a MicroVAX.

5) spooler of complete event data to other VAX/VMS computers in the network;

6) collector of the alarm conditions;

7) periodic monitoring of the apparatus performance.

The VMS system is composed of several parallel subprocesses that are scheduled according to a pre-determined scheme of priorities and are synchronized via event flags. Other multiple processes, substantially decoupled from the core of the system, are the user interfaces (e.g. consoles, histogram presenters). The data sharing among the processes is performed via mailboxes and global sections, while the raw data flow between the different components of the system is arbitrated by the model buffer manager [28], developed at CERN. The network service is performed by DECNET communications. The system implements synchronous DECNET transparent communications in the subprocess that routes the directives to a companion process of the ELN systems (e.g., send commands/wait for the completion) and asynchronous DECNET nontransparent communications in the subprocess that handles unsolicited data coming from the ELN systems (e.g., messages containing event data, alarms).

The data spooler tasks accept connections from the remote monitoring jobs and distribute on request raw data and information on the running conditions. The event display interactive programs are an example of a

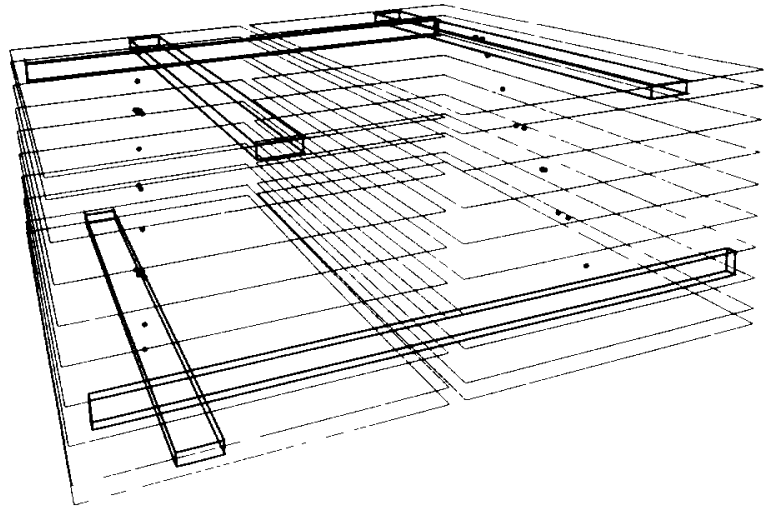

Fig. 27. Online event display of two muon tracks in the first supermodule. The rectangular boxes indicate scintillator counters hit and the points are streamer tube hits.

remote job receiving data from the data spoolers. The event display performs the 3D visual presentation of the events shown in fig. 27, using GKS [29]. Data sources for the event displays could be also the raw data files stored in the disks of the wide area network. The stellar collapse monitor is another example of a job receiving data from the data spoolers. This job executes a real-time monitor of possible signals due to a stellar collapse and sends alarms in case of candidate events.

The apparatus monitoring is performed via the histogram presenters, which make use of HBOOK [30] and ZEBRA [31]. They allow the presentation and the manipulation of the filled histograms. The histogram handler provides data to the local presenters via a global section and to remote presenters via a disk file storing a periodic dump of the histogram contents. A further stage of the monitoring is performed by a "post End-Run" program running in batch. The procedure makes available the raw data to several routines designed for the efficiency evaluation of the different components of the apparatus, for the book-keeping of the running conditions, pre-analysis procedures, etc. In the first supermodule of MACRO, the total number of electronic channels read was as shown in table 2 .

Table 2

\begin{tabular}{lcc}
\hline & Channels & $\begin{array}{l}\text { Max sıze } \\
{[\text { kbyte] }}\end{array}$ \\
\hline 1) Steamer tube system & 24000 (digital) & 3.5 \\
2) QTP system & 60 & 6 \\
3) Scintillators ERP, & & \\
$\quad$ ADC, TDC & 700 & 1.4 \\
4) Scintıllators WFD & 28 & 31 \\
5) PHRASE & 41 & 0.95 per \\
& & channel \\
\hline
\end{tabular}


Two microVAXes were used in the run with the first supermodule: one for stellar collapse (PHRASE) and the other for the muon physics and the monopole search. The MicroVAX for stellar collapse collected events at the rate of about $0.9 \mathrm{~Hz}$. An event was defined at the single counter level. The event length was about 0.95 kbyte. After the data reduction performed in the MicroVAX the event length was about 0.24 kbyte. The second MicroVAX collected data at a rate of about $0.13 \mathrm{~Hz}$. The average event length was 9 kbyte, with a maximum of about 34 kbyte. After the data reduction and compression the average event length was 3.2 kbyte. The average dead time was of the order $0.5 \%$. A VAXstation 3100 was used for event display and stellar collapse monitor, and the data were obtained using our network facilities. Data were logged on the VAX8200 disks and then periodically copied on the VAX8700 in the external laboratory.

\subsection{The universal time (UTC) clock}

The time of occurrence of the events is determined by a system (UTC clock) which has as the primary component a master clock, located in the external laboratory, driving peripheral units (slave clocks) located in the tunnel. The Master clock uses a high stability rubidium oscillator as a local frequency source. The measured long term frequency fractional drift, $\Delta \nu / \nu$, of the oscillator is less than $10^{-11}$. Two methods are used to align the clock to the universal time scale. The first, low quality, method was based on the statistical analysis over periods of 1 to 15 days of radio broadcast time signals. It gives an accuracy of about 0.1 ms. The second method used now is based on measuring the position, relative to the local time scale, of the television frame synchronization signals. The comparison with the same measurement performed on the same signals by the Italian time standard institution (Galileo Ferraris), relative to the UTC scale, allows the calculation of time corrections for interesting events with a residual discrepancy of $\sim 1 \mu \mathrm{s}$ relative to UTC, apart from a constant offset of up to $1 \mathrm{~ms}$ that is not yet measured. The time information is transported to the slave clocks in the underground experimental areas through monomodal optical fiber cables, $8 \mathrm{~km}$ long. A slave clock presents to the data acquisition system a pattern of 80 bits containing the time with $0.1 \mu \mathrm{s}$ resolution. Whenever an event triggers the apparatus, the output register of the clock is frozen to allow the reading operation (five CAMAC words of 16 bits each). An input/output register (CES IOR 1420) interfaces the clock to the CAMAC dataway. In order to reach a high degree of redundancy, the UTC clock system will be augmented in the final configuration by addition of a global positioning system (GPS) receiver. This will monitor the local time scale by means of time signals from satellite based standard clocks.

\section{Off-line data analysis environment}

Data recorded on the acquisition computer (VAX 8200) are processed through pass 0 of the off-line analysis program DREAM (Data Reduction and Event Analysis for MACRO). This pass simply reformats raw data files onto a multi-run tape, written in a machine independent format with the addition of some information about the run status in each run header. DREAM is a versatile program conceived to manage the different stages of MACRO analysis: data I/O (pass 0), event decoding (pass 1), reconstruction of calibrated physical parameters (pass 2), and event reconstruction (pass 3). It is written in standard FORTRAN 77 code using only general packages that are portable to different machines (mainly the CERN library). The data memory management is accomplished by the ZEBRA package, which allows both a dynamic storage of data structures and machine independent $\mathrm{I} / \mathrm{O}$ services. The portability of the program has been tested on VAX/VMS, IBM/VM and UNIX based IBM RISC /6000 computers. The complete analysis through pass 3 requires $\sim 0.15 \mathrm{~s} /$ event on a VAX 8600 . The calibration data from the scintillator system are collected at each run and during some special dedicated runs. The calibration database resulting from the analysis of calibration records and runs are stored on direct access files (RZ/ZEBRA) for the reconstruction of calibrated data (pass 2). The track reconstruction on the streamer tube hits is performed on independent views (wire, strip and vertical plane views) requiring the alignment of at least four hits for each track. The tracks reconstructed in the different views are then merged and space tracks are defined in the unambiguous cases. The tracking package takes care of the grouping of parallel tracks in the same event (multimuon bundles). Special care has been dedicated to the geometrical survey of the streamer tube system to have a good absolute reference system, as required by the muon astronomy analysis. These data are kept in a geometry database that is updated from time-to-time as more detailed surveys or more refined analyses become available.

Another important tool of the MACRO off-line analysis is a GEANT [32] based simulation program, GMACRO, which describes the experimental apparatus in all its details (geometry and detector responses). GMACRO is interfaced to many input event generators (multi-muon events, muons from cosmic point sources, monopoles, etc.) and produces both raw data and machine independent data samples with the same structure as real data. These data can be processed by 
a standard DREAM run, thus allowing the evaluation of the MACRO acceptance and the reconstruction program efficiency. Both DREAM and GMACRO include graphic event display facilities (developed using GKS and X-window graphic packages) for the visual analysis of complicated events. The calculation of the real path length of the reconstructed tracks in the rock overburden above the detector is done by using digitized data provided by the IGM (Istituto Geografico Militare) over a wide region $\left(10 \times 10 \mathrm{~km}^{2}\right)$ around the Gran Sasso laboratory. The elevation map used has a height step of $25 \mathrm{~m}$ in the regions with slope on average larger than $12 \%$, and of $5 \mathrm{~m}$ elsewhere. These data have been reduced using interpolation techniques and converted to standard rock depth as a function of the zenith and azimuth angles. Programs giving the muon survival probability for each rock thickness have also been developed.

\section{Acknowledgements}

We gratefully acknowledge Enrico Bellotti (Director, LNGS), the Istituto Nazionale di Fisica Nucleare, the Laboratori Nazionali del Gran Sasso, the US Department of Energy, the US National Science Foundation, the North Atlantic Treaty Organization, and the Fulbright Foundation for their generous support of the MACRO experiment.

\section{References}

[1] For a review of the theory of GUT monopoles, see, for example, J. Preskill, Ann. Rev. Nucl. Part. Sci. 34 (1984) 461.

[2] J. P. Petrakis et al., Nucl. Instr. and Meth. A268 (1988) 256.

[3] I.B. Berlman, Handbook of Fluorescence Spectra of Aromatic Molecules, 2nd ed. (Academic Press, 1971).

[4] See for example W.D. Arnett et al., Ann. Rev. Astron. Astrophys. 27 (1989) 629.

[5] R.J. Ducar and P.M. McIntyre, IEEE Trans. Nucl. Sci NS-26 (1979) 301.

[6] D.J. Ficenec et al., Phys. Rev. D36 (1987) 311.

[7] G.W. Foster, Ph.D. Thesis, Harvard University, 1983.
[8] F. Bourgeors, Nucl. Instr. and Meth. 219 (1984) 153.

[9] A. Baldinı et al., Nucl. Instr, and Meth. A305 (1991) 475

[10] G. Battistoni, Nucl. Instr. and Meth. A279 (1989) 137.

[11] G. Battistoni et al., Nucl. Instr. and Meth. A245 (1986) 277.

[12] G. Catanesı et al., Nucl. Instr. and Meth. A247 (1986) 438.

[13] E. Iarocci, Nucl. Instr. and Meth. 217 (1983) 30, and references therein.

[14] G. Battistoni et al., Nucl. Instr, and Meth. 176 (1980) 297.

[15] G. Battistoni et al., Nucl. Instr and Meth. 217 (1983) 429.

[16] S.D. Drell et al., Phys. Rev. Lett .50 (1983) 644.

[17] G. Battistoni et al., Nucl. Instr. and Meth. A235 (1985) 91.

[18] G. Battistoni et al., Nucl. Instr. and Meth. A270 (1988) 185.

[19] M. Ambrosio et al., Nucl Instr. and Meth. A301 (1991) 275

[20] E. Barbarito et. al., Nucl. Instr and Meth. A300 (1991) 581

[21] G Auriemma et al., Nucl. Instr. and Meth. A263 (1988) 249.

[22] S. Ahlen et al., Nucl. Tracks Rad. Meas. 19 (1991) 641; and Proc. 15th Int. Conf. on Particle Tracks in Solids, Marburg, Germany, eds. R. Brandt, R. Spohr and P. Valer (Oxford) p. 641.

[23] R. Fleischer, P.B. Price and R.M. Walker, Nuclear Tracks in Solids (California Press, Los Angeles, 1976) p. 18

[24] MACRO Collaboration, Proc. 14th Int. Conf. Solid State Nuclear Track Detectors, Lahore, Pakistan, 1988, ed. S.A. Durrani (Oxford) p. 331.

[25] MACRO Collaboration, Proc. XXI ICRC, vol. HE 7.2-10, pp. 252. Adelaide, Australia (1990); Nucl. Tracks Radiat. Meas. 19 (1991) 641.

[26] I. D'Antone et al., IEEE, Trans. Nucl. Scı. NS-36 (1989) 1602

[27] See for example A.M. Tenenbaum and M.J. Augenstein, Data structures using PASCAL (Prentice Hall, 1981).

[28] P. Vande Vyvre, The Model Buffer Manager User's Guide, CERN, Geneva, May 1987.

[29] GKS-GRAL Gmbh, GKSGRAL-3d User Manual, Darmstadt, May 1988.

[30] R. Brun and D. Lienart, CERN Program Library Y250, Geneva, CERN, October 1987.

[31] R. Brun and J. Zoll, CERN Program Library Q100, Geneva, CERN, January 1987.

[32] R. Brun et al., DD/EE/84-1, CERN, Sept. 1987. 